\title{
Federated Architecture for Secure and Transactive Distributed Energy Resource Management Solutions (FAST-DERMS) \\ System Architecture and Reference Implementation
}

\section{January 2022}

Fei Ding

Weijia Liu

Jason MacDonald

James Ogle

Annabelle Pratt

Avijit Saha

Joe Hagerman

Murali Baggu 


\section{Disclaimer}

This work was authored in part by the National Renewable Energy Laboratory, operated by Alliance for Sustainable Energy, LLC, for the U.S. Department of Energy (DOE) under Contract No. DE-AC3608GO28308. Funding provided by the U.S. Department of Energy Office of Energy Efficiency and Renewable Energy Building Technologies Office and the U.S. Department of Energy Office of Electricity's Advanced Grid Research Program through the Grid Modernization Initiative.

This work was authored in part by the Pacific Northwest National Laboratory, operated by Battelle for the U.S. Department of Energy under Contract DE-AC05-76RL01830.

This work was authored in part by the Lawrence Berkeley National Laboratory operated under Contract Grant No. DE-AC02-05CH11231.

This work was authored in part by Oak Ridge National Laboratory, managed by UT-Battelle LLC for the US Department of Energy under contract DE-AC05-00OR22725. 


\section{Federated Architecture for Secure and Transactive Distributed Energy Resource Management Solutions (FAST-DERMS)}

System Architecture and Reference Implementation

Fei Ding ${ }^{1}$

Jason MacDonald ${ }^{2}$

Annabelle Pratt ${ }^{1}$

Joe Hagerman ${ }^{4}$

\author{
Weijia Liu ${ }^{1}$ \\ James Ogle ${ }^{3}$ \\ Avijit Saha ${ }^{1}$ \\ Murali Baggu ${ }^{1}$
}

January 2022

\footnotetext{
${ }^{1}$ National Renewable Energy Laboratory

${ }^{2}$ Lawrence Berkeley National Laboratory

${ }^{3}$ Pacific Northwest National Laboratory

${ }^{4}$ Oak Ridge National Laboratory
} 


\section{Summary}

This document provides system-level specifications for a federated architecture for secure and transactive distributed energy resource management solutions (FAST-DERMS), presents a solution, and describes operational concepts for the proposed solution. FAST-DERMS enables the provision of reliable, resilient, and secure transmission and distribution (T\&D) grid services through the scalable aggregation and nearreal-time management of utility-scale and small-scale distributed energy resources (DERs).

We first present the principles and objectives of FAST-DERMS. Then, after discussing important system concepts, we present the specifications for FAST-DERMS and a solution that employs a distributed and federated control methodology in which the DERs connected to a single point of common coupling with the rest of the system, such as individual substations, are optimized coordinately to provide system-level grid services.

FAST-DERMS aims to aggregate and coordinate the operations of DERs to support T\&D grid operations. The key optimization and control component of this FAST-DERMS reference implementation is a flexible resource scheduler (FRS) that aggregates the DERs within a substation service area. These FRSs operate at the substation level and perform constrained economic dispatch of DERs, either directly or through a transactive market or aggregator, as shown in Figure ES-1. An FRS Coordinator at the distribution system operator (DSO) level aggregates distribution substations operated by FRSs and interfaces with the transmission system operator (TSO) to provide transmission services. FAST-DERMS also allows for the integration of the FRS Coordinator with an existing distribution utility management system that could be employed by the DSO to enhance distribution grid operations.

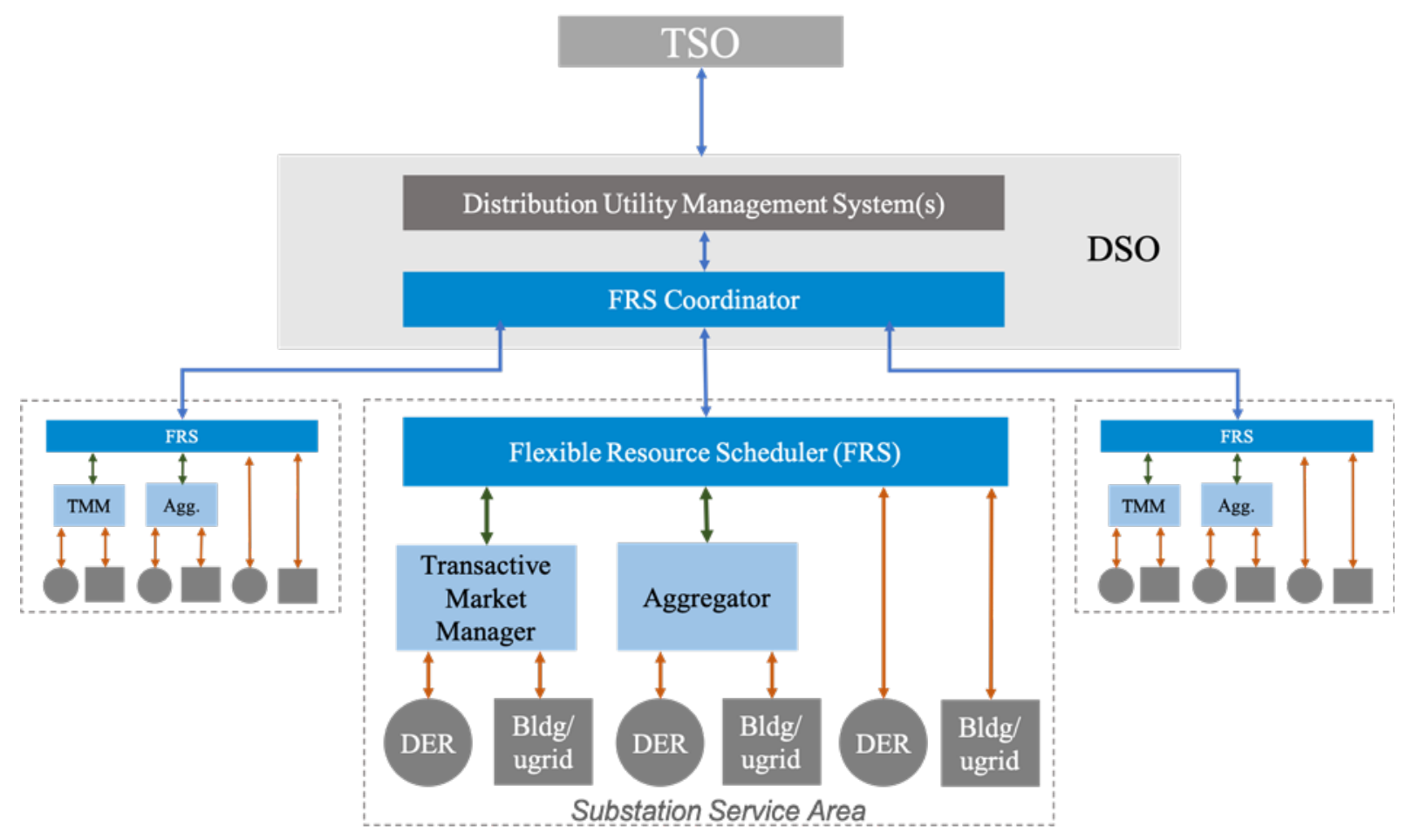

Figure ES-1. Conceptual diagram of FAST-DERMS

This FAST-DERMS reference implementation relies on several key components, including: 
- DERs: A broad range of DERs - including solar photovoltaics, battery energy storage systems, electric vehicles, building loads, and other distributed generators - is considered in the FASTDERMS design. These DERs and the local aggregations of these DERs can offer flexible and dispatchable active power and/or reactive power that can be used to support grid services.

- FRS: The FRS is the key optimization and control component of FAST-DERMS. Its main function is to generate firm offers to the wholesale market operated by the TSO through performing reliabilityconstrained commitment in coordination with distribution network management and dispatch of DERs connected to a substation. These FRSs operate at the substation level. DERs can subscribe, either directly or through an aggregator or transactive market, to the FRS and be treated as schedulable resources via different incentive options and at different aggregation levels.

- FRS Coordinator: The FRS Coordinator maps and aggregates distribution substations operated by individual FRSs to the appropriate pricing nodes in transmission markets.

- Aggregators: Third-party or utility-owned aggregators that already aggregate and control a subset of the DERs in the distribution network can also subscribe to the FRS and become controllable assets. The aggregator directly interacts with the FRS by providing an aggregated flexibility of its DERs. The operations of individual DERs are still managed by the aggregator.

- Transactive market: FAST-DERMS also recognizes a transactive energy market as one method to aggregate and implement DER controls. A transactive market manager is an important FASTDERMS reference implementation component. It manages transactive DERs through a subscription to the FRS.

Also, the successful implementation of DER management requires close collaboration with existing utility monitoring and management systems, such as distribution supervisory control and data acquisition systems, outage management systems, distribution management systems, and advanced distribution management systems. To maintain safe, reliable, and secure operations, utilities must provide an overarching authority to DER operations.

FAST-DERMS follows a "total DSO" approach to enable the interactions between DERs at distribution networks and TSOs. This approach requires all resources sited in a distribution network to be aggregated through the distribution utility or their surrogate, ensuring that the DSO maintains control of its network and enabling the DSO to manage any competing objectives between the needs of the T\&D systems. Specifically, FAST-DERMS provides a scalable and interoperable approach for distribution utilities to aggregate and control a variety of DERs to participate in wholesale electricity markets and provide transmission grid services. With the network-level stochastic optimization solved by the FRSs, FASTDERMS can manage the uncertainty in the flexibility offers from DERs within the distribution network, and thus they can provide a firm aggregate service to the transmission system.

Standard-based communications protocols will be used in FAST-DERMS to support reliable and interoperable data exchange. This document provides guidance for the communications architecture to manage complexity and to encourage interoperability in the context of the FAST-DERMS reference implementation. A separate communications system design document will specify the specific standards and profiles for implementation. This document provides a high-level overview of the standards appropriate for the interfaces among different FAST-DERMS components.

Given the increasing numbers and sophistication of cyber and physical threats, FAST-DERMS also incorporates approaches to detect anomalies and to provide a fail-safe to secure grid operations in the presence of cyber-physical attacks. This document provides a brief overview of these approaches. 


\section{Acknowledgments}

The authors thank Jason Autrey, Tom Bialek, Tom Eyford, Jesse Gantz, Andrew Ingram, Rahul Kadavil, Dino Lelic, Greg Smith, George Stefopoulos and Brad Williams for feedback on the draft of this document. We also thank Ryan Fedie, Erika Gupta and Chris Irwin of the U.S. DOE for their guidance.

The views expressed in the article do not necessarily represent the views of the DOE or the U.S. Government. The U.S. Government retains and the publisher, by accepting the article for publication, acknowledges that the U.S. Government retains a nonexclusive, paid-up, irrevocable, worldwide license to publish or reproduce the published form of this work, or allow others to do so, for U.S. Government purposes. 


\section{Acronyms and Abbreviations}

\begin{tabular}{|c|c|}
\hline ADMS & advanced distribution management system \\
\hline AMI & advanced metering infrastructure \\
\hline BESS & battery energy storage system \\
\hline BMS & building management system \\
\hline BTM & behind the meter \\
\hline CAISO & California Independent System Operator \\
\hline DER & distributed energy resource(s) \\
\hline DERMS & distributed energy resource management system \\
\hline DMS & distribution management system \\
\hline DNP3 & Distributed Network Protocol 3 \\
\hline DSO & distribution system operator \\
\hline DSSE & distribution system state estimation \\
\hline EMS & energy management system \\
\hline $\mathrm{EV}$ & electric vehicle \\
\hline FAST-DERMS & $\begin{array}{l}\text { Federated Architecture for Secure and Transactive Distributed Energy Resource } \\
\text { Management Solutions }\end{array}$ \\
\hline FoM & front of the meter \\
\hline FRS & flexible resource scheduler \\
\hline GIS & geographic information system \\
\hline GMLC & Grid Modernization Laboratory Consortium \\
\hline HEMS & home energy management system \\
\hline HVAC & heating, ventilating, and air conditioning \\
\hline IEC & International Electrotechnical Commission \\
\hline IEEE & Institute of Electrical and Electronics Engineers \\
\hline ISO & independent system operator \\
\hline MGC & microgrid controller \\
\hline MPC & model-predictive controller \\
\hline NERC & North American Electric Reliability Corporation \\
\hline OMS & outage management system \\
\hline PV & photovoltaic \\
\hline RTO & regional transmission organization \\
\hline $\mathrm{T} \& \mathrm{D}$ & transmission and distribution \\
\hline TMM & transactive market manager \\
\hline TSO & transmission system operator \\
\hline SCADA & supervisory control and data acquisition \\
\hline VAR & volt ampere reactive \\
\hline VPP & virtual power plant \\
\hline
\end{tabular}




\section{Contents}

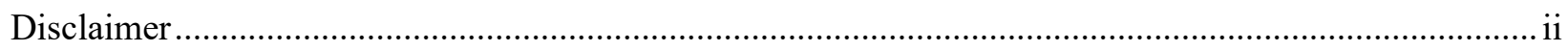

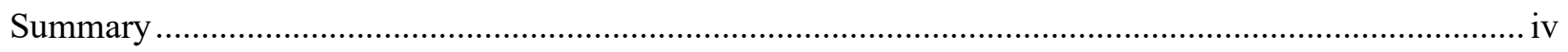

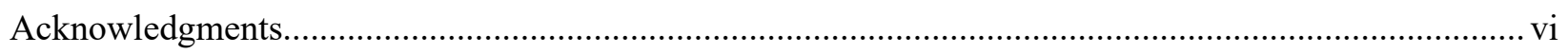

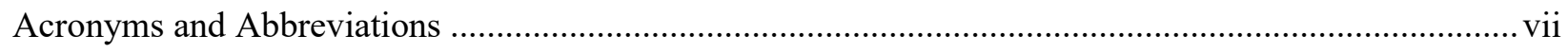

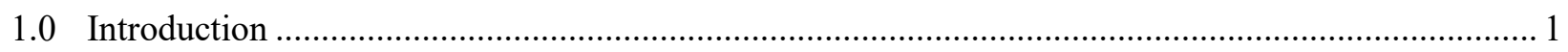

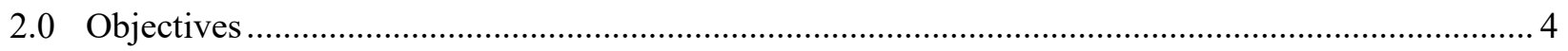

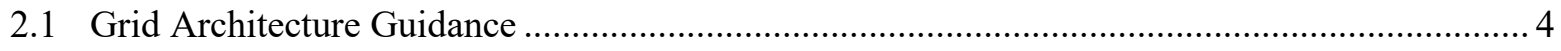

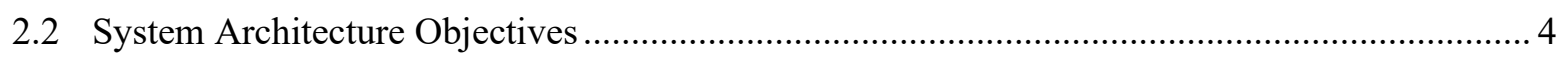

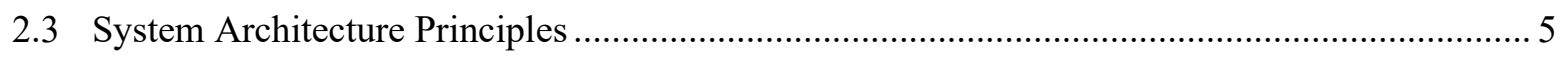

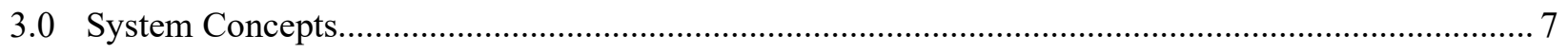

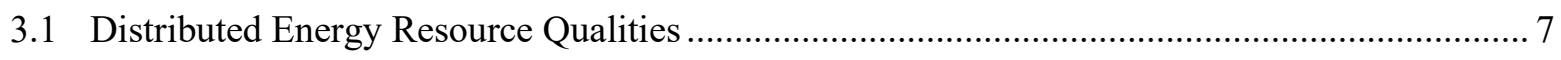

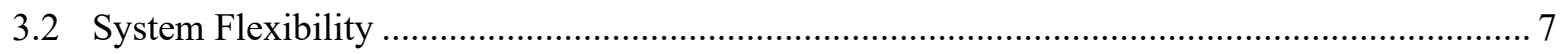

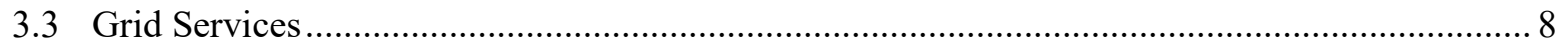

3.4 Distributed, Centralized, and Decentralized Systems ...................................................... 8

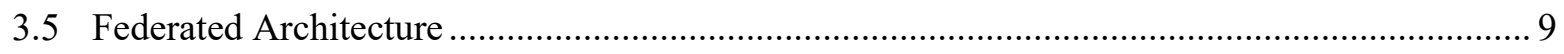

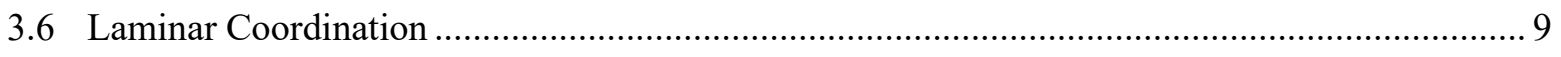

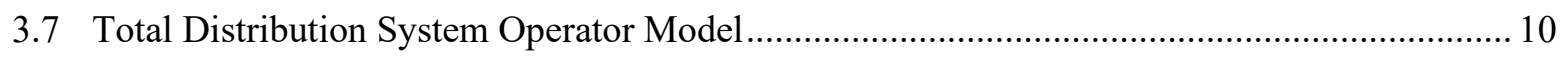

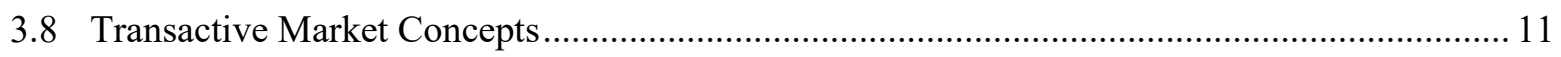

3.9 Secure Distributed Energy Resource Management for Grid Operations ................................. 12

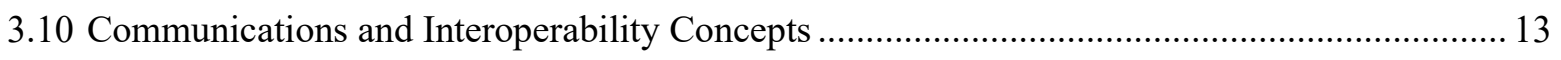

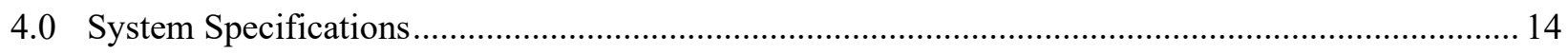

4.1 Distributed Energy Resource Flexibility Management in Total Distribution System Operator

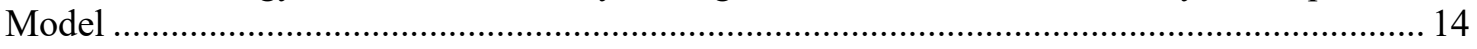

4.2 Federated Structure for Heterogeneous Distributed Energy Resource Types and Aggregation

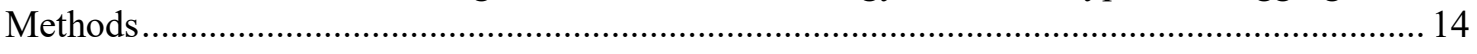

4.3 Distributed Energy Resource Management with the Laminar Coordination Framework .......... 15

4.4 Secure Distributed Energy Resource Management for Grid Operations ................................ 15

4.5 Communications and Interoperability .......................................................................... 15

5.0 Federated Architecture for Secure and Transactive Distributed Energy Resource Management

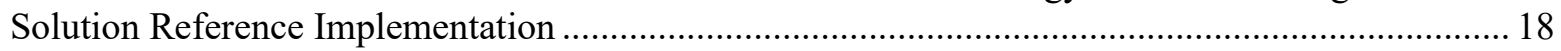

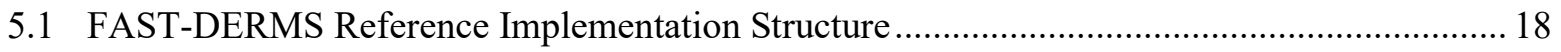

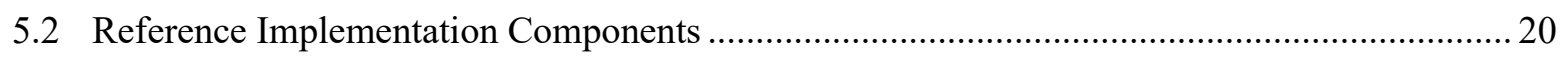

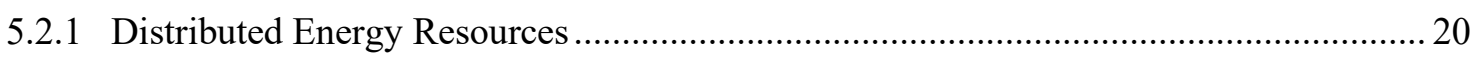

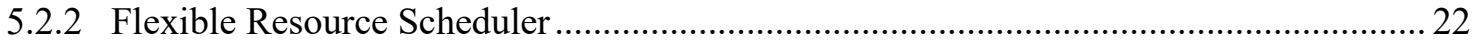

5.2.3 Flexible Resource Scheduler Coordinator.................................................................. 22

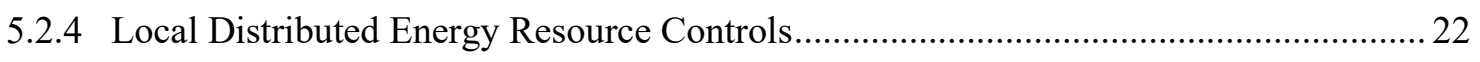

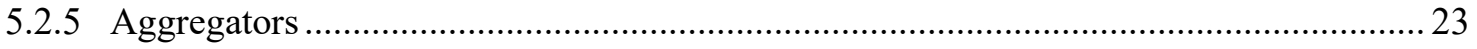




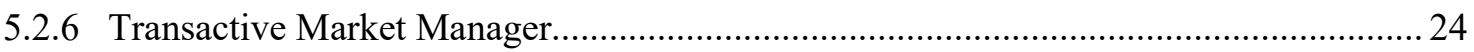

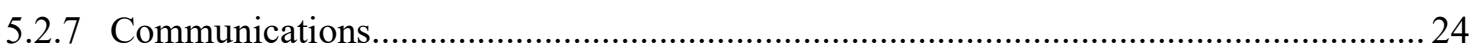

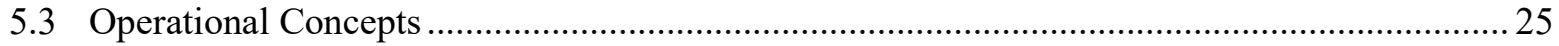

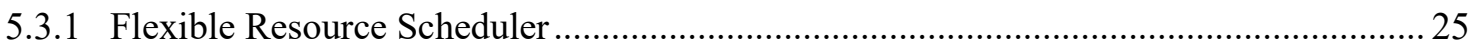

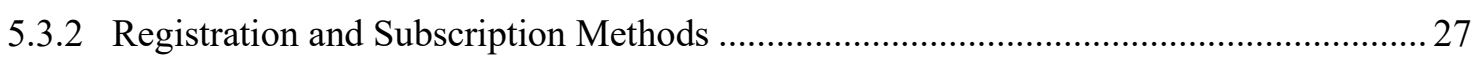

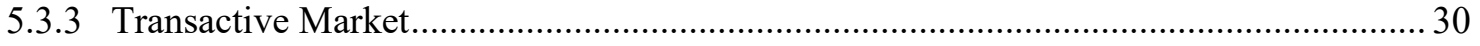

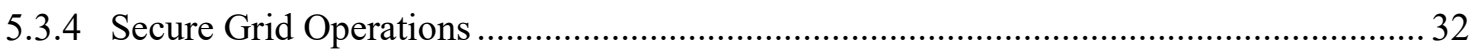

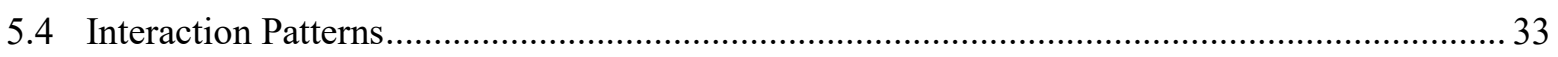

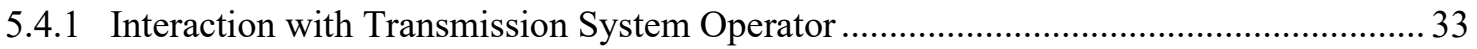

5.4.2 Interactions with Existing Distribution Utility Systems ............................................... 34

5.4.3 Interactions with a Wholesale Market Operated by a Transmission System Operator ... 36

5.4.4 Interactions within a Traditionally Regulated Market Environment............................... 39

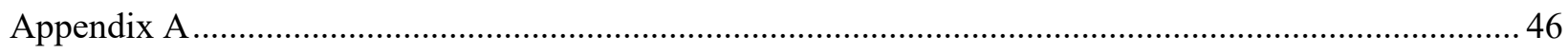

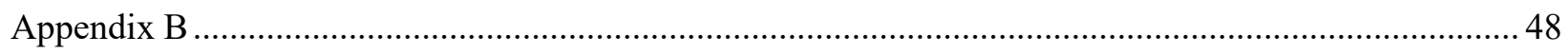

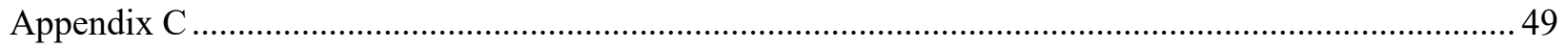

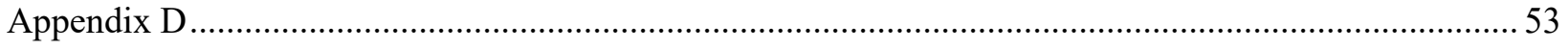




\section{Figures}

Figure 1. FAST-DERMS manages a wide variety of DERs to provide grid flexibility ...................... 3

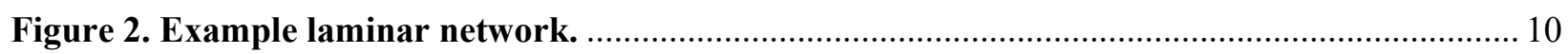

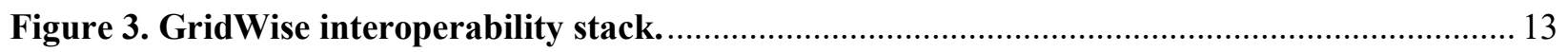

Figure 4. High-level FAST-DERMS reference implementation structure ….................................. 18

Figure 5. Structure of the FAST-DERMS reference implementation................................................ 19

Figure 6. FAST-DERMS reference implementation communications interfaces ............................ 25

Figure 7. Transactive energy market based on one-way communication......................................... 31

Figure 8. Transactive energy market based on two-way communications ........................................ 32

Figure 9. A two-step process adopted in the FAST-DERMS reference implementation to secure grid

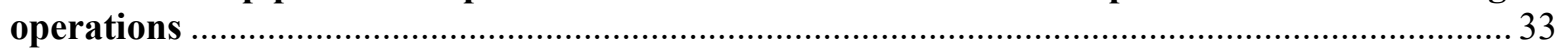

Figure 10. Responsibilities of the FRS and scheduling coordinator based on the market timeline .. 34

Figure 11. FRS process flow to participate in wholesale markets .................................................. 37

Figure 12. Conceptual diagram of FAST-DERMS applied to a vertically integrated utility in a traditionally regulated market environment .................................................................. 40 


\subsection{Introduction}

Traditionally, transmission grid services have been provided by power generating plants, and distribution grid management is achieved by legacy voltage regulating devices, such as transformer on-load tap changers, capacitor banks, and voltage regulators. As the capacity of distributed energy resources (DERs) increases rapidly in modern distribution grids, DERs have become significant contributors to reshaping the generation mix and the energy consumption profiles; therefore, using DERs to provide energy and grid services at both the transmission and distribution (T\&D) levels has started to be investigated by utilities and related stakeholders and has been identified as a promising area for research, development, and demonstration (Sun and Frew 2021).

There are various definitions of DERs - some limit the DERs to distributed generation sources (NERC 2017), and some consider only behind-the-meter (BTM) DERs (DNV GL 2014). In this project, we employ a general and extended definition: a DER can be a local generation unit, such as rooftop solar photovoltaic (PV) and fuel cells; a storage unit, such as a battery; a flexible and controllable load with demand response capability, such as an electric vehicle (EV); or a schedulable building load located at low- to medium-voltage distribution systems (International Renewable Energy Agency 2019b) (California Public Utilities Commission 2020a). The following key features of DERs can be identified based on the adopted definition:

- A DER can act as a power producer, a power consumer, or even a prosumer that can switch its role at will. For some DERs (e.g., inverter-based PV and batteries), active power and reactive power can be controlled separately.

- DERs can be very different from each other in terms of rated power and capacity, consumption pattern, generation profile, control method, operational constraints, etc.

- DERs can provide flexibility because they are controllable within certain physical limitations, such as power and capacity limits. Some DERs - e.g., BTM building loads that directly influence the comfort of end users - are subject to additional constraints to meet customers' preference.

The growth in DERs has brought both challenges and opportunities to utilities (Ardani, O'Shaughnessy, and Schwade 2018). Proper cost-effective management of DERs can provide valuable services to meet the demands of electric grids and customers. At the same time, utilities must deal with challenges, including conductor or equipment overload, protection device malfunction caused by reverse power flow, voltage violations, limited real-time communications, retail rate limitations, and cross subsidies. So far, utilities have investigated different DER integration and coordination schemes to tackle these challenges. Several examples include:

- Xcel Energy introduced demand response programs for customers with controllable loads, such as heating, ventilating, and air-conditioning (HVAC) devices and industrial freezers (Xcel Energy 2016). Economic rewards are provided to incent customers to reduce electricity consumption during peak demand hours.

- Hawaiian Electric offered a fast demand response scheme that employs DERs to help maintain the stability of the grid (Hawaiian Electric 2014). The responses of DERs can be either completely automated (where control signals will be directly sent from the utility operator) or semiautomated (where customers will manually respond to demand response requests). Owners of DERs participating in the demand response scheme receive financial incentives.

- Pacific Gas and Electric Company implemented a field demonstration of a distributed energy resource management system (DERMS) in managing DERs (Ardani, O'Shaughnessy, and Schwade 2018). The 
demonstrated DERMS use cases include DER data visualization, overload mitigation, voltage regulation, DER price response, and operational flexibility improvement.

Numerous optimization and control strategies have been developed for different types of DERs over the years; however, DER management and control are still siloed and at times conflicting between various DER technologies and the distribution management systems. Centralized control methods are widely adopted to manage large-scale DERs, but a centralized control structure is not scalable to manage a vast number of DERs. On the other hand, BTM DERs are generally managed according to local control objectives, and they are poorly integrated with the rest of the grid assets. To summarize, the existing DER control and management methods have the following limitations:

- Utilities generally do not have real-time or near-real-time visibility of DERs, especially BTM DERs; hence, the observability of the distribution grid is reduced.

- Existing DERs are typically managed by either a centralized controller or according to local control objectives. A large number/capacity of DERs acting only in response to local objectives could lead to operational issues in distribution grids, such as voltage sags and feeder overloads.

- Most DERs are associated with uncertainty factors, including weather conditions and customer behavior; however, DER uncertainty has not been well addressed in existing studies. Also, more accurate DER modeling techniques are required to accommodate DER integrations with various types and characteristics.

- Existing DER controls typically interact with the local distribution grid for services such as peak demand management and volt/volt ampere reactive (VAR) optimization. On the other hand, some commercial aggregators have managed to collect and control DERs to directly participate in bulk grid services while bypassing the distribution-level control. This can lead to conflict between transmission-level market and distribution operational considerations. New mechanisms are needed to integrate dispersed DERs and interact with both the distribution system and the transmission system to provide holistic grid services.

To address these limitations, this document specifies a federated architecture for secure and transactive DER management solutions (FAST-DERMS). FAST-DERMS enables the provision of reliable, resilient, and secure T\&D energy and grid services through the scalable aggregation and near-real-time management of utility-scale and small-scale DERs. FAST-DERMS addresses the recent FERC Order 2222 (Department of Energy Federal Energy Regulatory Commission 2020) by providing an architectural solution to enable DERs at the distribution system to participate in wholesale markets. It encompasses the management of a broad range of DERs, including PVs, battery energy storage systems (BESSs), EVs, flexible building loads, combined heat and power, and other distributed generators, as shown in Figure 1. These DERs can be dispatched directly if they act individually; as part of a fleet consisting of a single technology, such as an EV fleet; or as part of a local aggregation of different types of DERs in buildings, managed by a home energy management system (HEMS) or building management system (BMS), or among buildings, managed by a microgrid controller (MGC). 


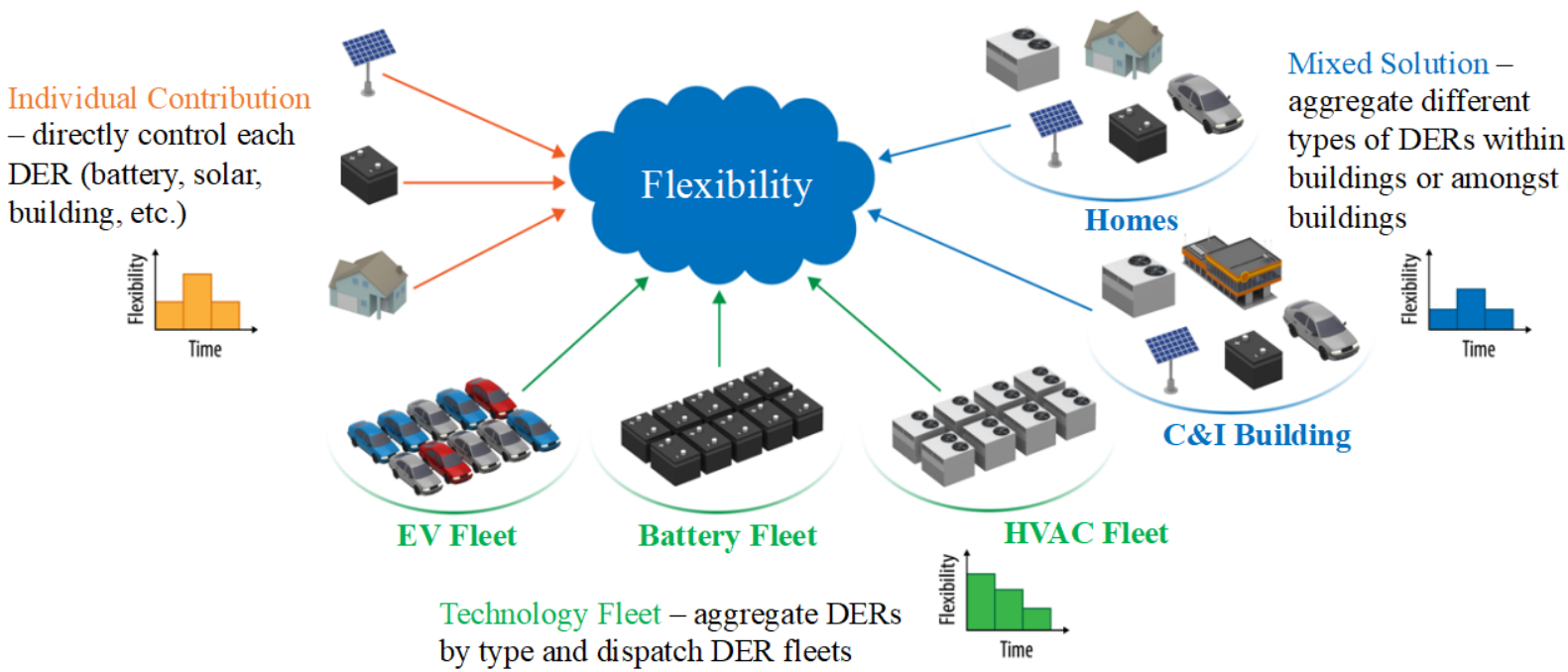

Figure 1. FAST-DERMS manages a wide variety of DERs to provide grid flexibility

Increased DER penetration has also driven the power industry to reconsider the role of distribution utilities, and the concept of a distribution system operator (DSO) is gaining ground (Rahimi and Mokhtari 2014). Although not many utilities in the United States today are considered DSOs, we envision that DSOs will become common in the future, and therefore we present the proposed FAST-DERMS with reference to DSOs. There is a spectrum of organizational models for DSOs. Instead of covering all features or possible definitions, we assume only the following key functions for a DSO: (1) responsible for real-time operations of the distribution system, (2) operates an open and transparent distribution market; (3) manages DERs; and (4) performs long-and short-term planning (Black \& Veatch Management Consulting 2020). 


\subsection{Objectives}

\subsection{Grid Architecture Guidance}

The FAST-DERMS system architecture is using the guiding principles from the Grid Modernization Laboratory Consortium (GMLC) Grid Architecture project (GMLC 1.2.1) ${ }^{1}$. This body of work examined the limitations of the existing grid relative to the desired qualities of a modernized grid and specified new grid structures to enable the realization of those desired qualities.

Architecture specifications relevant to the objective of enabling the grid to accommodate and use DERs were consolidated into a summary document, referred as the "reference grid architecture document" (Taft and Ogle 2021). This document identified several architectural objectives:

- Provide industry structure models that relieve constraints on the use of DERs, facilitate the increasing penetration of DERs, and enable their use for customer and system benefit.

- Accommodate DERs that might exist in dynamic heterogeneous mixtures that vary across a system with multiple models of DER use, ownership, and operation.

- Enable improved electric system reliability and resilience by automating grid operations and responses.

- Enable creative uses of and access to DER assets, and enable new business models for the grid.

- Expand bulk power system and distribution system relationships to improve system operation and resilience.

- To the extent that the grid structural changes can enable, support, or provide potential to improve recovery after some failure, they should do so.

The reference grid architecture document also provides specifications for a grid with high penetrations of DERs. FAST-DERMS is specifically aligned with a subset of these specifications as outlined in Appendix D. See the reference grid architecture document (Taft and Ogle 2021) for more detailed discussions of these specifications. The reference grid architecture document contains additional specifications that are not required for FAST-DERMS but with which FAST-DERMS is compatible.

The grid architecture specifications provide structures at a grid level. It informs the system and components architecture to ensure that the complex set of components and relationships form a cohesive whole at the grid level.

In the following sections, this document provides system architecture principles and objectives, followed by important system concepts. Then we present system-level specifications. Finally, we present a description of a FAST-DERMS reference implementation, including operational concepts.

\subsection{System Architecture Objectives}

The fundamental goal of FAST-DERMS is to enable the provision of reliable, resilient, and secure T\&D energy and grid services through the scalable aggregation and near-real-time management of utility-scale and small-scale DERs. To achieve this goal, the system architecture has been developed to support the following objectives:

\footnotetext{
${ }^{1}$ https://gmlc.doe.gov/projects/1.2.1.
} 
- Enable the provision of energy and grid services at the T\&D level from utility-scale and small-scale DERs.

- Coordinate reliability-constrained economic dispatch of DERs connected to a distribution system to balance customer and system benefits. Resulting efficiencies enable reduced cost of service for all customers.

- Provide a mechanism, such as stochastic optimization, to manage the uncertainty in the flexibility that can be provided by DERs within the distribution network, and thus to provide a firm aggregate service to the transmission system.

- Transparently integrate with existing distribution utility management systems and allow for flexible system deployment.

- Transparently integrate with a broad range of DER types, including solar PVs, BESSs, EVs, building loads, and other distributed generators.

- Enable the coordination of DERs under utility or nonutility ownership/operation and coexistence of different control schemes for DERs.

- Encompass different aggregation levels, including single entities and different kinds of aggregations, including fleet operators that aggregate the same types of DERs (e.g., EVs or batteries) and aggregators that aggregate different types of DERs (e.g., commercial and industrial curtailment service providers).

- Enable transactive markets to manage the aggregated performance of a group of DERs. Market-based signals such as electricity price may be employed to coordinate the DER energy transactions in the distribution grid. Both passive responses (such as DERs responding to locational marginal pricing or dynamic pricing signals) and active flexibility provisions (such as DERs submitting bid prices for energy transactions) need to be considered.

- Detect and protect against increasing numbers and sophistication of cyber and physical threats. Incorporate approaches to detect anomalies and provide a fail-safe to secure grid operations in the presence of cyber-physical attacks.

- Consider the impact of DERs that do not participate in the coordination/control solutions provided by the DSO, such as treating them as inflexible net load on the system. For example, some nonutilityowned resources could be operated or managed by themselves and not participate in DSO services. Because they do not directly interact with the DSO, these resources are both uncontrollable and present high uncertainty to the DSO.

\subsection{System Architecture Principles}

The following principles were core considerations in the development of the FAST-DERMS system architecture.

- Follow a total DSO model, as described in Section 3.7, where all resources connected to the distribution level that participate in transmission markets are orchestrated through the DSO to enable interactions between DERs at distribution networks and TSOs. This approach requires all participating resources sited in a distribution network to be aggregated through the distribution utility or their surrogate, ensuring that the DSO maintains control of its network and enabling the DSO to manage any competing objectives among the needs of the T\&D systems.

- The coordination of DERs must respect grid operational constraints. To maintain safe, reliable, and secure operations, utilities must provide an overarching authority to DER operations; therefore, DER 
aggregation and control should be achieved through close interactions between the DER aggregation mechanisms and existing distribution utility management and communications systems.

- Apply Laminar Coordination Framework principles (See Section 3.6) to manage the complexity of the coordination of increasing numbers of DERs and varying forms of aggregation while enabling customer and system benefits. Distribute the control of DER flexibility optimization to substation and lower layers to manage at scale.

- Apply standards-based communications interfaces between system components in a layered communications architecture separating functionality from data integration to avoid dependencies with specific deployments. 


\subsection{System Concepts}

This section provides general descriptions of concepts that are key for FAST-DERMS, including DER qualities, system flexibility, grid services, distributed control, federated control, laminar coordination, the total DSO model transactive markets, secure DER management and communications and interoperability. Entity definitions are provided in Appendix A.

\subsection{Distributed Energy Resource Qualities}

There are various definitions of DERs - some limit the DERs to distributed generation sources (NERC 2017), and some consider only BTM DERs (GL 2014). In this project, we employ a general and extended definition: a DER can be a local generation unit, such as rooftop solar PV and fuel cells; a storage unit, such as a battery; a flexible and controllable load with demand response capability, such as an EV; or a schedulable building load located at low- to medium-voltage distribution systems (International Renewable Energy Agency 2019b) (California Public Utilities Commission 2020a). The following key features of DERs can be identified based on the adopted definition:

- A DER can act as a power producer, a power consumer, or even a prosumer that can switch its role at will. For some DERs (e.g., inverter-based PVs and batteries), active power and reactive power can be controlled separately.

- DERs can be very different from each other in terms of rated power and capacity, consumption pattern, generation profile, control method, operational constraints, etc.

- DERs can provide flexibility because they are controllable within certain physical limitations, such as power and capacity limits. Some DERs - e.g., BTM building loads that directly influence the comfort of end users - are subject to additional constraints to meet customers' preference.

DERs can be clustered according to their basic characteristics as a load, generator, or storage (New York ISO 2017). Loads can only withdraw energy from the grid and can provide services such as demand response and demand shifting. Typical loads include building loads and EVs without vehicle-to-grid capability. Generators can only inject energy into the grid and can participate in energy markets and potentially provide ancillary services, such as regulation and reserve. The most common generation-only resource in distribution systems is solar PV. A BESS is an ideal example of a storage resource because it can both withdraw energy from and inject energy into the grid. Storage resources can provide many grid services, including those mentioned and others, such as peak demand management, congestion management, and frequency regulation.

\subsection{System Flexibility}

The increasing integration of variable renewable energy resources in T\&D grids has brought significant uncertainty and intermittence to power systems in terms of planning and operation. As indicated by the famous "duck curve" (Loutan 2015), more resources are required to provide services (e.g., ramping and reserve) to maintain system reliability to cope with the sharp changes in the system net load curve (U.S. Department of Energy 2020a); hence, flexibility becomes an important and desired attribute of modern power systems.

The GMLC Foundational Metrics Analysis project (U.S. Department of Energy 2020a) defines flexibility as "the ability to respond to future uncertainties that might stress the system in the short term and require the system to adapt over the long term." In this report, we focus on short-term flexibility in the 
operational domain. FAST-DERMS aims to enable DERs to improve power system flexibility through various aggregation and control methods.

In general, power system flexibility can be evaluated in the following three dimensions (U.S. Department of Energy 2020a):

- Overgeneration/curtailment: Excess generation from renewable energy sources could result in curtailment because of power system reliability requirements. The ability to reduce overgeneration (i.e., curtail generation) is a key indicator of flexibility.

- Ramping: Larger ramping capacity, reduced ramping cost, and faster ramping speed can more effectively handle the fluctuations caused by intermittent renewable energy generation and load variation, resulting in a more flexible power system.

- Uncertainty: Generation and load variations will result in oscillations and deviations of system parameters such as frequency and voltage that could affect power system reliability. Systems with higher flexibility can withstand a higher volume of fluctuations caused by uncertainties associated with both generation and demand.

\subsection{Grid Services}

The key functionality of a power system is to deliver consistent and reliable power to customers. Because the nature of electricity demands that power generation and consumption must be balanced in real time, meeting customer expectations is challenged by various factors, such as supply-demand variation, device malfunction, component failure, and cyber threats; therefore, different types of grid services have been promoted to tackle these issues from different perspectives and to help maintain electricity service quality.

Grid services can be clustered into two categories: transmission grid services and distribution grid services. In general, transmission grids and distribution grids are very different in terms of scale, capacity, voltage level, and control method. More information on these services is summarized in Appendix C.

In addition to transmission grid services, FAST-DERMS enables DERs to enhance local distribution grid performances in terms of reliability and power quality. The improved distribution grid performances will benefit DER owners in return.

\subsection{Distributed, Centralized, and Decentralized Systems}

To be consistent with the grid architecture reference specification (Taft and Ogle 2021), we use the following definitions:

- A decentralized system is one in which the elements are separate (usually geographically dispersed but not always) and act independently, with perhaps some small amount of supervision to provide set points, etc.

- A distributed system is a decentralized system in which the elements cooperate to solve a common problem. This implies some form of communication among the decentralized elements.

- A centralized system is one in which all the computing, logic, control, data analysis, etc., is performed at a single element. 
Distributed systems might have a central element that participates in the overall processes, which is sometimes characterized as a hybrid of central and distributed architectures.

The modern electric grid comprises centralized, distributed, and decentralized elements both in the physical grid structure as well in the control structure.

\subsection{Federated Architecture}

A federated architecture is a pattern that allows for interoperability and information sharing between semiautonomous, decentralized control systems and applications. In this context, a federate is a collection of DERs that are aggregated and managed by some control paradigm - for example, a transactive market or a building management system that manages DERs as well as loads.

Federated systems typically have some form of consolidation and services layer on top of decentralized elements (e.g., a federated data warehouse or federated network). A federated network lets multiple networks (which could be geographically distributed) work together by employing shared services and a central management framework for consistent configuration and policies.

\subsection{Laminar Coordination}

The basic structural elements that are used to compose laminar networks are discussed in (Taft and Ogle 2021). Typically, a master problem is decomposed into secondary problems, and an inter-domain communications bus facilitates bidirectional information flow between coordinator nodes. Multiple decompositions are possible for complex problems, with multiple coordination domains. These elements provide building blocks for assembling laminar networks for a specific grid or a portion of a grid. Each laminar node requires intelligence, i.e., computing capacity.

An example of a laminar network is shown in Figure 2. The coordination root node could be extended to the independent system operator (ISO)/regional transmission operator (RTO), but it does not need to be because of the roles and responsibilities defined for the total DSO model, discussed in Section 3.7. 


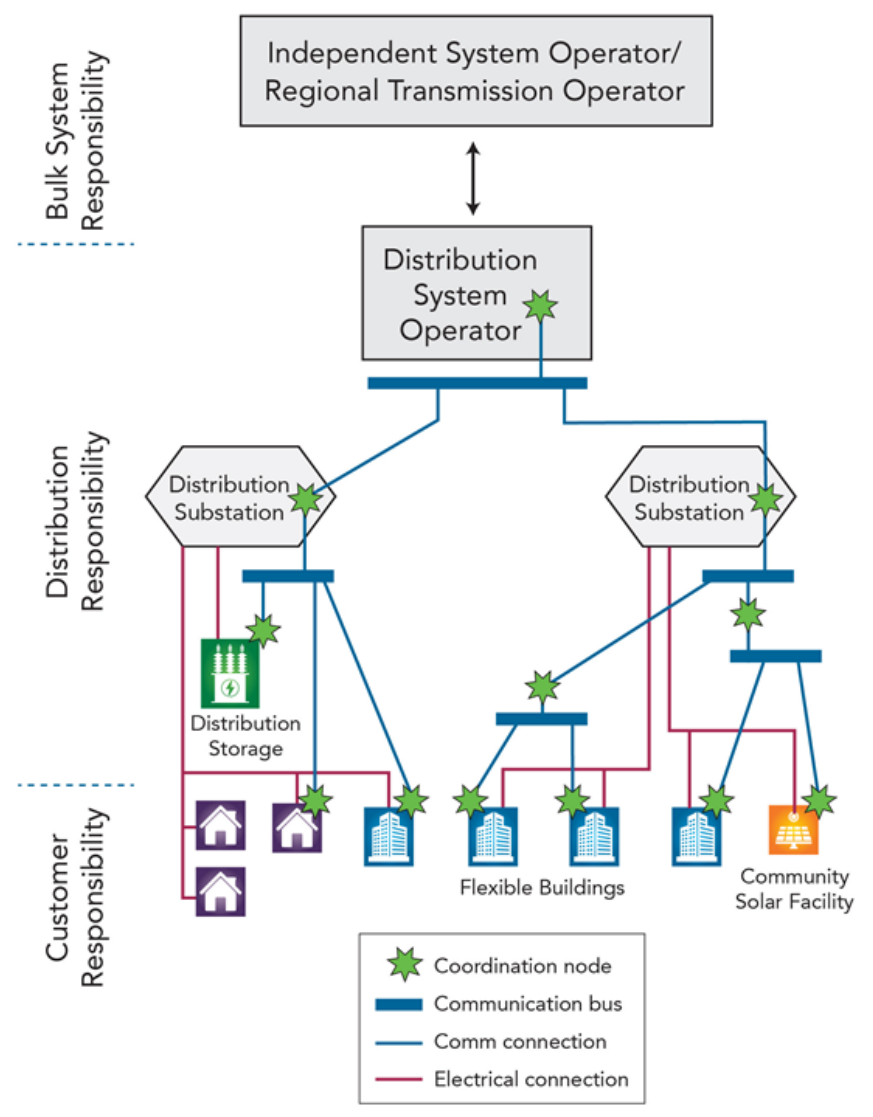

Figure 2. Example laminar network. Image from Interop Strategic Vision Whitepaper (March 2018)

\subsection{Total Distribution System Operator Model}

Traditionally, distribution systems are modeled as lumped substation nodes in a transmission system, and the distribution system is treated as a pricing node in the locational marginal pricing markets. With the increasing proliferation of DERs, however, more and more DERs are aggregated and participating in wholesale markets. This brings new requirements for defining the roles of TSOs and DSOs. The California Independent System Operator (CAISO) has identified a spectrum of possible designs that can be envisioned in terms of the complementary roles of DSOs and TSOs (Kristov 2014).

FAST-DERMS is following the total DSO approach-i.e., the DER aggregators operating resources in a distribution system should go through the DSO to participate in the wholesale market. A TSO only optimizes the transmission system with the distribution network modeled as an aggregated P-node, ${ }^{1}$ and the DSO is responsible for dispatching all resources in the distribution network and ensuring distribution system reliability, efficiency, resilience, and security. There should be no information passing from the distribution network to the transmission network or vice versa, other than the data exchange between the TSO and DSO, resulting in a more scalable and secure TSO-DSO interaction paradigm. It is structurally sound and simpler to implement (Kristov 2014), and it can provide a clean interface and separation of roles and responsibilities for DSOs and TSOs (Taft and Ogle 2021). The total DSO model enables the

\footnotetext{
${ }^{1}$ In organized markets using the locational marginal pricing market paradigm, the transmission-distribution interface substation is a pricing point, often called a "pricing node" or "P-node."
} 
TSO to use distribution-connected assets for grid resilience and operational flexibility purposes without interfacing with distribution operations or impacting distribution system reliability.

\subsection{Transactive Market Concepts}

The transactive energy approach is widely considered as a promising solution to coordinate a large number of dispersed, decentralized DERs through an incentive-based mechanism. The most significant feature in this approach is that the DER control and coordination is achieved through the exchange of value-based information with explicit meaning to negotiate and, ultimately, settle on a price that best satisfies each party's needs and/or constraints.

Transactive energy has been implemented and tested in numerous pilot projects in the United States, most of which focus on:

- Developing control methodologies (e.g., how controls change verse price)

- Managing the system through pricing, including:

- Price formation: determining the first price or "seed" price

- Price discovery: determining the capacity or response from an offered price

- Price negotiation: determining the increase or decrease in price and response in an orderly way to encourage settlement.

- Evaluating the response from the approach (e.g., observing what happened or measurement/verification of what happened).

For example, the AEP gridSMART project introduced a real-time market to control DERs that were located BTM, such as HVAC systems (Widergren, et al. 2014) (Prinsloo, Mammoli and Dobson 2017). The Pacific Northwest Smart Grid Demonstration Grid project implemented and evaluated transactive energy concepts for a future smart grid (Lian, et al. 2017) (Agalgaonkar and Hammerstrom 2017). In most of these prior works, the DSO "scheduled" DERs based on local data through a price signal to implement transactive energy control (Abrishambaf, et al. 2019). A transactive energy system provides benefits to multiple parties of an energy system:

1. Distribution utilities want specific DER responses at a specific time (e.g., temporarily) and in a specific location (i.e., spatially) in the distribution system because they require services to correct for excursions and constraints or to avoid maintenance, defer capital upgrades, or manage other financial considerations of the utilities.

2. Consumers want DER and BTM assets to reduce their cost and to improve their services when (i.e., temporarily) and where (i.e., spatially) they can benefit — otherwise, the customer would do nothing and hold the utility to their (regulated) responsibilities.

3. Implementers, such as demand-side management providers or third-party energy service providers (e.g., energy contractors such as those that reduce demand charges or optimize for tariffs), want a structure where they can benefit or share in the value of the avoided costs.

In all these cases, price formation, price discovery, and price negotiation are critical to elicit responses in flexibility and energy use from disparate, decentralized devices to satisfy the utility constraints or needs and provide customer benefits. 
In any structure of transactions among multiple parties, buyers and sellers must manage their objectives, goals, and decision criteria; their financial or energy constraints; and their willingness or ability to exchange services compared to other options.

For any transaction, the market maker serves as the party responsible for ensuring that there is fairness in the exchange between the parties (or, more simply, a structure to transact openly and fairly). They do not, however, define the objectives and goals, the value perception for each party, or the economic utility of the transaction; rather, they help form the processes and settlement of the exchange. For example, the objective or primary goal of each participant or stakeholder is, notionally, described as follows:

- Because distribution utilities are responsible for reliability by managing and balancing a system with two-way power flows, distribution utilities might want new options to minimize price from their generation and transmission provider, to adhere to voltage constraints (e.g., $\pm 5 \%$ from the ANSI voltage tolerance boundary (ANSI C84.1)), to recruit voltage support, or to recruit supplemental reactive power.

- Consumers or implementers (such as demand-side management providers or third-party energy service providers) might want new options to minimize price or differentials in price between present contracts and future contracts, to improve comfort (e.g., reduce set point excursions), or to better manage other needs or expectations.

\subsection{Secure Distributed Energy Resource Management for Grid Operations}

FAST-DERMS relies on advanced operational technology and information technology to achieve enhanced DER monitoring and control capabilities; however, both cyber and physical threats are increasing in frequency and sophistication. FAST-DERMS is facing two major vulnerabilities to cyberphysical threats: misfunction of controls and communications interruption between the controls and DERs/DER aggregations. To address these vulnerabilities and achieve resilience against such attacks, effective approaches to detect anomalies and provide a fail-safe to secure grid operations in the presence of cyber-physical attacks are needed.

Many anomaly detection methods exist, and they can be divided into model knowledge-based and datadriven methods (Tan, et al. 2020). A critical function of distribution system state estimation (DSSE) is to detect, identify, and eliminate bad measurement data. The analysis is essentially based on the properties of residuals, and this is the most common model knowledge-based anomaly detection method. The weighted least squares estimation assumes that the measurement errors follow a Gaussian distribution. Using the linear relation between the residuals and errors, the mean and covariance of the measurement residuals can be derived after the DSSE process, and then the properties of the residuals (e.g., normalized distribution) can be used to formulate a test to identify the bad measurement data. In traditional weighted least squares DSSE, the detection of bad data is done only after the estimation by processing the measurement residuals. Based on the properties of residuals, only one suspicious measurement can be identified and then removed (or corrected) in each iteration. Then the DSSE must be processed repeatedly to get a correct estimation. On the other hand, data-driven methods typically use machine learning algorithms to build a model or map a relation of the studied system. If system data do not conform to the expected relations, then an attack can be identified. 


\subsection{Communications and Interoperability Concepts}

Given the scope of the FAST-DERMS problem domain, spanning from the TSO to the DSO interface through group-level aggregation, and individual DER connections, there is a complex industry-standard landscape. Various standards have been used in this space, including Distributed Network Protocol 3 (DNP3) (IEEE 1815) and the Institute of Electrical and Electronics Engineers (IEEE) Standard 2030.5. The latter has been selected as the choice for California implementation in Rule 21 (California Public Utilities Commission 2020b). Both communications standards can support different information models, and the Rule 21 DER information model is based on International Electrotechnical Commission (IEC) Standard 61850 and SunSpec Modbus.

The communications landscape gets even more complex if considering the various types of communications network technologies and associated protocols that could be used in a specific deployment. For the purposes of the FAST-DERMS communications architecture, the primary interoperability focus is at the information level, as defined by the GridWise Architecture Council's interoperability framework, depicted in Figure 3 (GridWise Architecture Council 2008). This layer provides a consistent semantic understanding or the domain definition of the information exchanged. In addition, it includes the business context to define how the information is applied to specific business process interactions. Category 6, the Business Procedures layer, is also relevant to ensure that the rules of the interactions are commonly understood. The IEC Common Information Model collection of standards (IEC-61970 and IEC-61968) contains examples of industry standards in these layers.

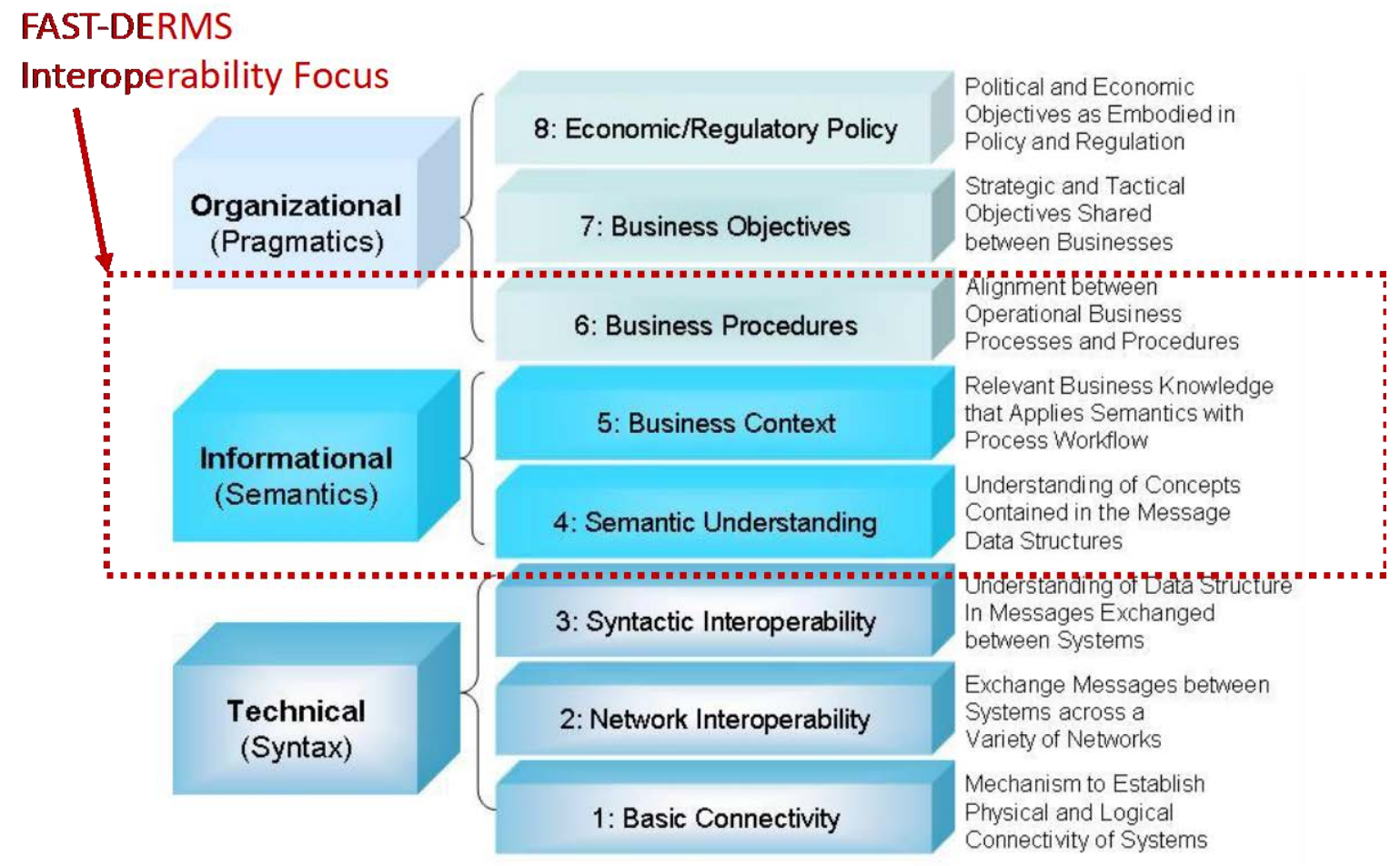

Figure 3. GridWise interoperability stack. Image from (GridWise Architecture Council 2008) 


\subsection{System Specifications}

This section provides the system specifications for FAST-DERMS.

\subsection{Distributed Energy Resource Flexibility Management in Total Distribution System Operator Model}

The reference grid architecture document (Taft and Ogle 2021) specifies several requirements for grid entities to support the preferred total DSO model presented in Section 3.7. In this structure, the DSO is responsible for traditional distribution system reliability management and for managing distribution assets to meet power and energy flows at the T\&D interface, to optionally participate in wholesale markets, and to provide grid services to the TSO. In addition, DER aggregators and other third-party energy service providers do not bypass the DSO to directly participate with the TSO.

Although this grid structure addresses challenges associated with potential tier bypassing, it creates additional responsibilities for traditional grid operators and scalability concerns because the number and variety of DERs within a distribution area can be large, as can the different aggregations methods. FASTDERMS provides a means by which the DSO can help manage that complexity while leveraging the benefits of DERs and maintaining traditional grid reliability management.

1. Specification 1:

1.1. The FAST-DERMS reference implementation shall conform to the TSO and DSO responsibilities as defined in the total DSO model.

1.2. A distributed flexibility management approach is responsible for coordinating the operation of the DERs across the entire distribution network to achieve the maximum operational flexibility for enhancing grid operations.

1.3. The distribution utility management system(s) responsible for managing the distribution system shall provide reliability constraints to the flexibility management function coordinating DER operations.

\subsection{Federated Structure for Heterogeneous Distributed Energy Resource Types and Aggregation Methods}

The distribution system might be subject to a broad range of DERs, including PV, BESS, EVs, flexible building loads, combined heat and power, and other distributed generators. These DERs might be managed individually or as part of a fleet consisting of a single technology, such as an EV fleet; as part of a local aggregation of different types of DERs in buildings managed by a BMS or by a HEMS; or among buildings managed by an MGC. DERs that are geographically dispersed and of varying types might be aggregated and managed via an aggregator. Such a condition presents a complex management front to the DSO responsible for the operation of the distribution system with these diverse DER interconnections and management paradigms.

To address this complexity, FAST-DERMS applies the federated architecture concept, described in Section 3.5, recognizes the coexistence of different aggregation methods of DERs, and enables the cooptimization and coordination of these different DER aggregations. In this regard, a federate is a collection of DERs that are aggregated and managed by some control paradigm - for example, a transactive market or a BMS that manages multiple DERs. A FAST-DERMS reference implementation 
must provide a framework for interoperability and information sharing by which each federate can participate with the DSO to provide flexibility in the form of energy and grid services. From the DSO perspective, the different forms of DER types, aggregations, and optimization objectives in the federates do not need to be exposed; they only need to see an interface by which to leverage potential flexibility services. By the same token, the federate does not need to know the details of the grid constraints that can drive service requests.

2. Specification 2:

2.1 Apply the federated architecture concept and incorporate different methods of aggregating DERs to enable co-optimization and coordination.

2.2 Include price-based controls as an aggregation method.

\subsection{Distributed Energy Resource Management with the Laminar Coordination Framework}

A FAST-DERMS reference implementation should follow a Laminar Coordination Framework, as described in Section 3.6.

3. Specification 3:

3.1 Assemble the coordination and control structure to support grid-constrained DER flexibility management from layered control domains.

3.2 Support distributed DER coordination minimally to the substation level.

3.3 Ensure extensibility to further distributed decomposition.

3.4 The types of resources within a control domain should be transparent to other layers.

\subsection{Secure Distributed Energy Resource Management for Grid Operations}

A FAST-DERMS reference implementation should address vulnerabilities to cyber-physical threats. In particular, the malfunction of controls and communications interruptions are the two major threats that a FAST-DERMS reference implementation should prepare for and have the capability to mitigate.

4. Specification 4:

4.1 Be aware of threats - for example, incorporate effective approaches to detect anomalies.

4.2 Have a fail-safe to secure grid operations in the presence of cyber-physical attacks.

\subsection{Communications and Interoperability}

The use of industry-standard communication protocols and profiles for FAST-DERMS is critical to achieving the desired interoperability objectives. FAST-DERMS does not dictate a particular standard provided it meets the necessary requirements for the information and behavior of the FAST-DERMS control architecture.

Similarly, the FAST-DERMS communications architecture does not specify any specific physical network technologies and should be compatible with network topologies and technologies that can provide the appropriate communications connections and quality of service necessary for FAST-DERMS operations. This is likely to be a mix of utility private communications networks and leased commercial service provider networks. In general, distribution supervisory control and data acquisition (SCADA) systems, where they exist, have a hub-and-spoke structure, generally using communications networks owned by the utility itself. This hub-and-spoke arrangement is augmented in a laminar coordination-based 
architecture, with communications networks and message buses at the feeder level aggregated at the substation level for local distributed controls. With increasing levels of nonutility-owned DER connections and potential energy service providers, there might be increasing use of the Internet and cloud services (Taft, De Martini and Kristov, 2015).

The communications architecture is guided by the grid architecture and FAST-DERMS principles, objectives, and specifications. The decision to follow a total DSO model in FAST-DERMS defines specific roles for the TSO and DSO that establish expectations and requirements for interactions between the two. In this model, the DSO is responsible for managing distribution assets. The DSO might provide services to the TSO based on DER flexibility in their area over the TSO-DSO interface. DER telemetry flows and dispatch instruction flow through the DSO layer, and the devices' specific details and control are not exposed to the TSO. This structure is summarized in the recommendations for DER telemetry in Taft (2017). Additionally, the TSO does not bypass the DSO to interact directly with DER devices, aggregators, or other energy service organizations.

FAST-DERMS coordinates DERs to provide energy and grid services but must be guided by the operational constraints of the distribution system to ensure that system reliability is maintained. To accomplish this, there must be information sharing between the DER management solution components and other utility management systems, such as an advanced distribution management system (ADMS), as well as a common representation of the distribution network model. A communications architecture that uses standards-based information models and separates data integration from application functionality within the DSO level will facilitate interoperability and allow flexibility and extensibility (Melton, et al. 2018).

A FAST-DERMS reference implementation must support the coordination of DERs connected to various feeders across the distribution system, owned or managed by different parties, and organized into a variety of different groupings or aggregations. At the same time, it must be aware of the structure and operational constraints of the distribution system network to ensure reliability is maintained. The communications architecture is guided by the grid architecture Laminar Coordination Framework concepts described in Section 3.6. The communications architecture must support multiple layers of laminar coordination. These can exist at the DSO, substation, aggregator, building/microgrid, and ultimately the DER device levels and the interface between the DSO and TSO. The primary communications interfaces occur between these layers (interlayer), but communications between elements within a given layer (intra-layer) are compatible with the architecture. This allows FAST-DERMS to be extensible with the future evolution of distributed controls for distribution system operation.

5. Specification 5:

5.1 Standards-based communications should be employed at all interfaces.

5.2 The information exchanged between the TSO and DSO for DER management should be limited to that needed for energy or grid services at the relevant T\&D interface point.

5.3 Information exchange approaches that separate data integration from application functionality should be used to facilitate interoperability and allow flexibility and extensibility.

5.4 Communications between multiple control domains is required. These can exist at the DSO, substation, aggregator, building/microgrid, and the DER device levels and the interface between the DSO and TSO.

5.5 The communications system should be compatible with distributed control approaches that require peer-to-peer communications within a layer. 
5.6 The FAST-DERMS reference implementation should not be dependent on fixed network topologies or technologies. It should be able to adapt to dynamic DER grouping and changes in network topology. 


\subsection{Federated Architecture for Secure and Transactive Distributed Energy Resource Management Solution Reference Implementation}

This section describes a specific FAST-DERMS reference implementation, including its overall structure, key components, and operational concepts.

\subsection{FAST-DERMS Reference Implementation Structure}

FAST-DERMS aims to aggregate and coordinate the operations of DERs to support T\&D grid operations. The FAST-DERMS reference implementation follows Specification 1, which requires a total DSO approach-i.e., the DER aggregators operating resources in a distribution system should go through the DSO to participate in the wholesale market. As described in Figure 4, the FAST-DERMS reference implementation leverages DERs - both individual DERs and local aggregations of DERs such as within a building (Bldg) or microgrid (ugrid) - to provide energy and grid services and to reduce the cost of service for all customers.

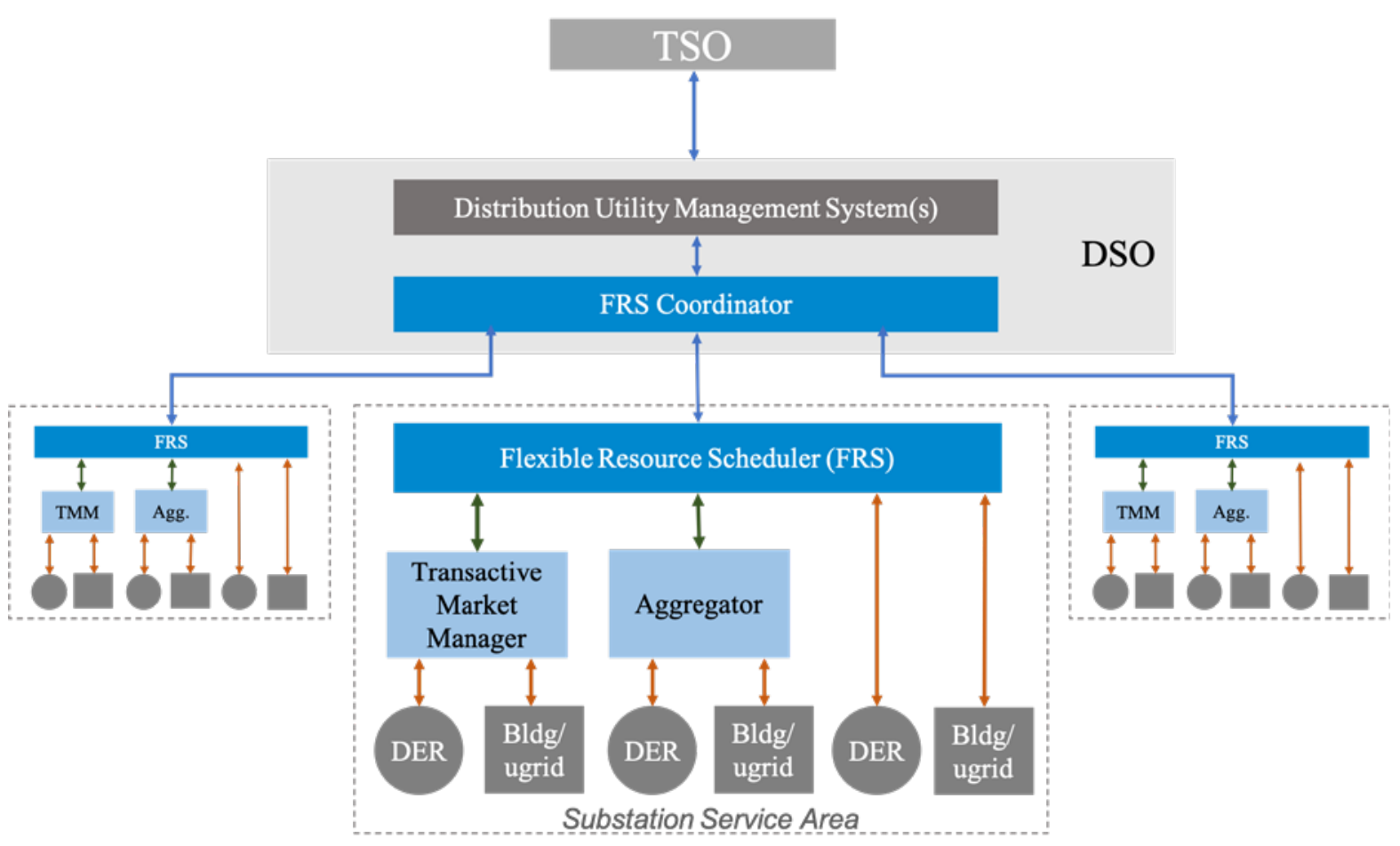

Figure 4. High-level FAST-DERMS reference implementation structure

The core functional component of the FAST-DERMS reference implementation is a flexible resource scheduler (FRS) that operates at the substation service area level to perform reliability-constrained economic dispatch of a heterogeneous set of DERs that might be aggregated by different means. These include local aggregations in buildings or microgrids, a DER aggregator, or a transactive market manager (TMM), thereby adhering to Specification 2. The FAST-DERMS reference implementation employs a distributed control methodology in which the resources downstream of a given substation are optimized independently with limited oversight from central distribution utility management system(s) that manage 
system reliability. This distributed approach can be further decomposed into various layers in the grid structure to form a self-similar hierarchical pattern consistent with the grid architecture Laminar Coordination Framework of Specification 3. The distributed approach also helps manage the scalability for the DSO. Multiple FRSs exist for a typical distribution system with multiple substations, and therefore an FRS Coordinator is needed to represent the overall distribution system at the DSO level.

The distribution utility management systems might offer constraints to the FRSs through the FRS Coordinator as well as request distribution grid services from the FRSs to support reliable distribution operations. The FRSs will coordinate the flexibility of DERs based on these constraints and distribution reliability. Bounded by constraints from the distribution operations, the FRS Coordinator can also facilitate DERs participating in the TSO wholesale market; thus, the FRSs and FRS Coordinator become core components of a DSO's operational systems, complementing existing reliability management responsibilities to help manage the complexity of coordinating high levels of DERs.

Finally, the FRS Coordinator interfaces - either directly or through an existing distribution utility management system or scheduling coordinator - with the TSO to negotiate the provision of energy and transmission grid services.

Figure 5 shows a more detailed view of the solution structure with many different kinds of DERs, including both front-of-the-meter (FoM) DERs (such as utility-scale PV power plants, utility-scale BESS, and public EV charging stations operated by utilities) and BTM DERs (such as rooftop PVs, small BESS, light-duty EVs, and controllable loads that are located at commercial and residential buildings).

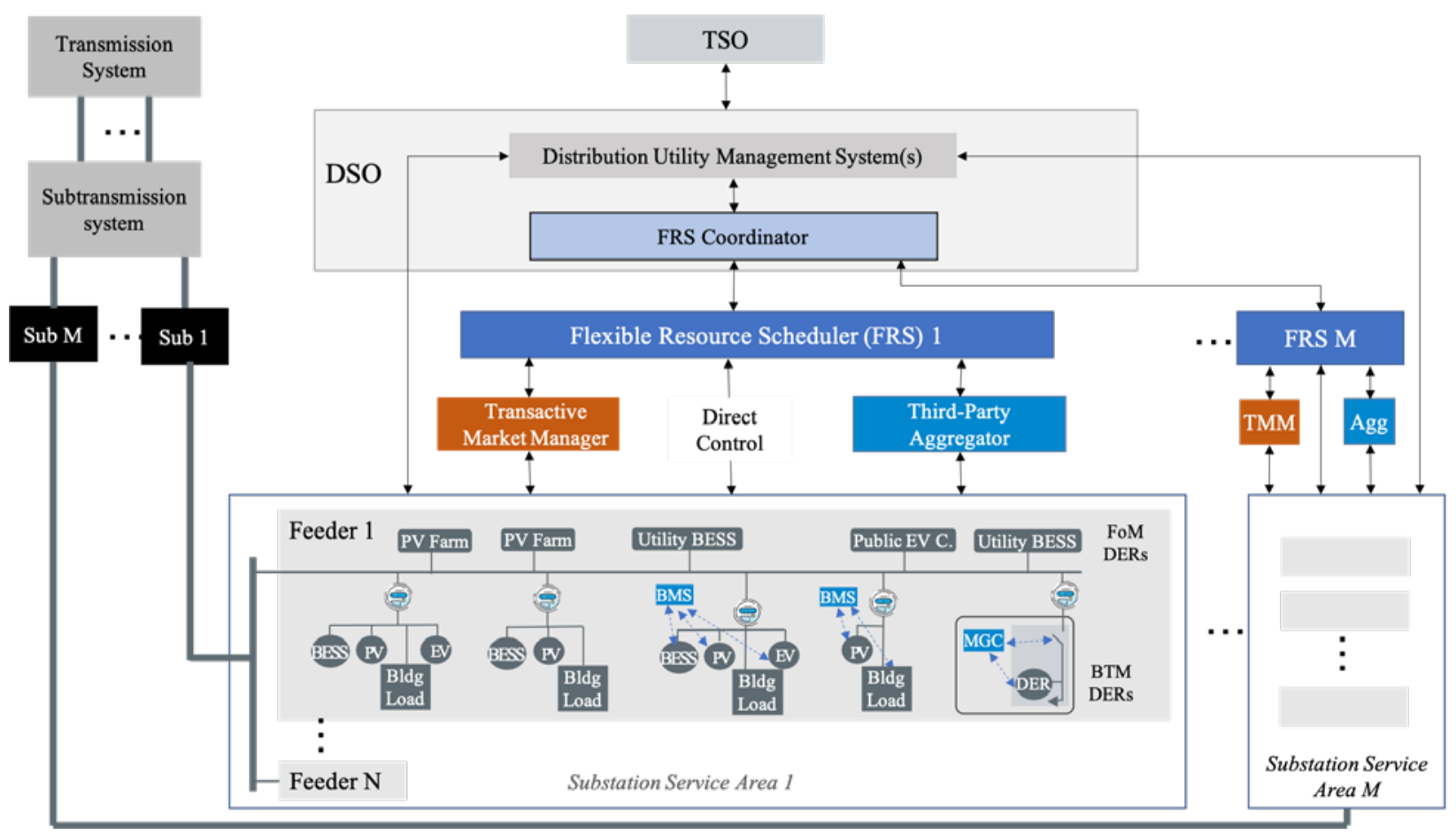

Figure 5. Structure of the FAST-DERMS reference implementation

Each FRS manages the DERs located downstream of a substation (Sub-1 through Sub-M). The FRSs and FRS Coordinator can be either stand-alone systems or integrated with existing distribution utility management systems. In either case, the FRSs will provide a way for the DSO to manage DERs in the entire distribution network to acquire the maximum operational flexibility for enhancing grid operations. If a utility management system exists - such as a DMS, an outage management system (OMS), or an 
ADMS - it is still monitoring and controlling legacy devices (such as voltage regulators) and some DERs (such as utility-owned BESS).

DERs can subscribe directly to the FRS and participate as individuals or as part of an aggregation managed by an entity such as a BMS, MGC, aggregator, or a TMM. Entities such as a BMS or MGC that manage local aggregations of DERs can subscribe directly to the FRS or through an aggregator or transactive market, allowing for multiple levels of aggregation. Any subscriber to the FRS - either an individual DER or an aggregating entity - chooses the type of incentive they receive and indicates the degree of aggregation they belong to. This allows for resources that can respond to real-time changes in price signals as well as those that prefer a capacity payment and opt for direct control.

FAST-DERMS provides a scalable approach to integrating DSO-managed DERs into wholesale electricity markets and transmission system operations following the total DSO model. This model requires that all resources sited in a distribution network are aggregated through the distribution utility or their surrogate, ensuring that the DSO maintains control of its network and enabling the DSO to manage any competing objectives between the needs of the T\&D systems (Kristov 2014). We recognize, however, that some resources in the DSO's service territory could be operated by a third-party DERMS and interface directly with the TSO. Although this introduces the risks that are addressed by the total DSO model, the FAST-DERMS reference implementation can offer some mitigation of these risks. This special case is discussed in Section 5.3.2.3. In brief, the impact of DERs that do not subscribe to the FRS or are not managed by a DMS/ADMS that interacts with the FRS will be incorporated into the uncertainty modeling in the FRS.

Communication is an important element in FAST-DERMS to support reliable information exchange among different components. The FRS requires information from its subscribers to solve its optimal scheduling problem. The details and specifics of the information depend on the subscription method. For instance, under direct control, it is expected that the FRS could have access to all types of DER information, including both static information (such as DER locations and capacities) and time-varying DER information (such as real-time DER flexibility and availability). But if a DER is participating via an aggregator or a TMM, the FRS might only have knowledge of the DER location and capacity and will likely not have access to real-time DER dispatch information that is determined by the internal control decisions made by the aggregator or TMM. The FRS also needs to send its scheduling decisions to the subscribed resources, and the scheduling decision will be further disaggregated and communicated to individual DERs. Data exchanges among the DERs, aggregators, FRSs, TSO, and other distribution utility management systems are also necessary to realize the features and functions of FAST-DERMS. All the information flow will be realized via standards-based communications protocols, with appropriate augmentation to support the advanced probabilistic features of the architecture.

\subsection{Reference Implementation Components}

This section provides a description of the key components of the FAST-DERMS reference implementation. Entity definitions are provided in Appendix A.

\subsubsection{Distributed Energy Resources}

In this project, we consider a general case where many DERs with distinct characteristics and different control approaches exist in the distribution system. Examples of the DERs we consider are shown in Figure 8, including FoM DERs and BTM DERs. Generally, a BTM DER is privately owned and operated by customers, and it provides power that can be used on-site without passing through a meter. BTM DERs are typically small in capacity (e.g., $<100 \mathrm{~kW}$ ). On the other hand, an FoM DER provides power to 
off-site locations and passes through an electric meter before reaching end users. They typically have large capacities (e.g., $>100 \mathrm{~kW}$ ) and are owned or operated by distribution utilities. Various FoM DERs and BTM DERs can be found in existing distribution systems, and the following DERs are considered in the FAST-DERMS architecture:

1. FoM DERs:

- PV power plant: A PV power plant refers to a group of solar panels with a common point of connection. A PV power plant is connected to the distribution feeder through power inverters that can be controlled by the DSO.

- Utility-scale BESS: A utility-scale BESS consists of one or a group of battery energy storage unit(s). A utility BESS is connected to the distribution feeder through power inverters that can be controlled by the DSO.

- Public EV charging station operated by utilities: Public EV charging stations are typically located at commercial areas such as shopping centers and can host multiple EVs. Here, we consider public EV charging stations operated by utilities.

2. BTM DERs:

- Building BESS: A building BESS is a battery-based energy storage system installed at a residential or commercial building, and it typically serves as an emergency power supply. A BESS is connected to a building load supply system through its power inverter.

- Rooftop PV: Rooftop PV generally refers to solar panels installed on the rooftop of residential and commercial buildings. PV panels are connected to the building load supply system through power inverters.

- Building load: Building loads can consist of a variety of demands, such as lighting demand, air conditioning, and water heating.

- Light-duty EV: Light-duty EVs such as personal vehicles plugged into EV supply equipment available at residential buildings and commercial charging stations.

In addition, local aggregations of different DERs, such as at buildings or in microgrids, are also considered.

Both FoM and BTM DERs vary in their capabilities to provide grid services; thus, different DERs are suited to provide specific grid services according to the service requirements summarized in Appendix C. One aspect of capability is the frequency and speed at which the DER can react. As reviewed in Appendix $\mathrm{C}$, different grid services require different response speeds. Most DERs can respond to a limited number of grid service calls within a certain period. This is common in existing demand response (load-shedding) schemes. Some DERs can respond to service calls on an hourly basis. Energy service provision in dayahead and hour-ahead markets are good examples in which DERs respond to market dispatches by shifting their generation/consumption at these timescales. Finally, some DERs can be frequently called upon and are capable of promptly responding to grid service requests. The representative grid service that requires DERs to be very responsive is frequency regulation. Typical DERs that can provide frequency regulation services include inverter-based DERs, such as PV and BESS. Most building loads and other non-inverter-based DERs have much larger time constants and thus are not well suited to provide frequency regulation.

Further, grid services include active power-based services (such as frequency regulation) and reactive power-based services (such as voltage regulation). Transmission grid operations are generally more focused on active power, including on energy markets and ancillary services, such as demand response, frequency regulation, and congestion management. In distribution grids, peak load management mainly 
relies on active power management. Both FoM and BTM DERs can provide active power-related services. Inverter-based DERs, such as BESS and PV, can adjust both their active and reactive power generation/consumption; therefore, they are widely used to provide both active power-based grid services and reactive power-based grid services. Some building loads also produce reactive power, but their reactive power is typically not controllable or schedulable, so building loads are generally not used to provide reactive power-based grid services.

\subsubsection{Flexible Resource Scheduler}

The FRS determines the level of wholesale market interaction in FAST-DERMS. The focus of FASTDERMS is on wholesale market contexts run by an ISO or RTO that delivers electricity through competitive market mechanisms, though Section 5.4.4 includes discussion of FAST-DERMS within the context of traditionally regulated markets. ${ }^{1}$ Each FRS aggregates all DER types located downstream of a substation in a physical distribution network and computes an aggregated bid or schedule for the substation service area's net load (and in rare instances, net generation) for wholesale electricity markets as well as bids or schedules for any capacity-based ancillary services and other nonmarket services. The bids computed reflect any physical constraints on the distribution system such that all bids will result in voltages and equipment loading that is safely within operating limits. These bids are provided to the FRS coordinator to be combined and sent to the wholesale markets. Each FRS also disaggregates grid control signals in real time to the various resources subscribed to the FRS to meet the service objectives for the network. These transmission-level service offers/responses are accomplished while respecting the voltage and thermal constraints of the distribution system that the resources are connected to. The interactions among the FRSs, FRS coordinator, and the TSO are further explained in sections 5.4.1, 5.4.3, and 5.4.4.

The geographic scope of an FRS is the feeders connected to one substation. If computationally feasible, the FRS will co-optimize all feeders connected to a substation so that the substation tap changers and transformer thermal constraints can be considered. If it is not feasible, the feeders will be assumed to be independent of one another, and the FRS will treat each feeder as a separate resource connected to the substation bus. The FRS will also coordinate with an existing utility DMS, ADMS, or DERMS through the FRS coordinator to collect real-time measurements, topology, and utility equipment set points; to ensure that the outcomes behind the managed substation are not causing challenges elsewhere in the distribution system; and to collect DER schedules if some resources within the scope of the FRS are controlled by such systems, as described in Section 5.4.2.

\subsubsection{Flexible Resource Scheduler Coordinator}

The FRS Coordinator maps and aggregates distribution substations operated by individual FRSs to the appropriate pricing nodes in transmission markets.

It functions as an aggregator of independently optimized substations so that they can be bid into wholesale markets, and it directs the appropriate TSO dispatch and control signals to each FRS. It can represent each FRS as an independent market resource or as an aggregated resource.

\subsubsection{Local Distributed Energy Resource Controls}

DERs have local controllers with different control strategies, such as deadband control and droop control, depending on the characteristics of DERs and the nature of the control variables. FAST-DERMS does not

\footnotetext{
${ }^{1}$ As defined in the Federal Energy Regulatory Commission's Energy Primer: A Handbook of Energy Market Basics, June 2020, available at https://www.ferc.gov/sites/default/files/2020-05/energy-primer.pdf.
} 
require specific implementations of local DER controls; however, local DER controllers (such as for individual battery and PV inverters) and controllers for local DER aggregations (such as HEMS, BMS, and MGC) can choose to subscribe to the FRS. These local DER aggregations are generally too small to have the option to directly participate in a wholesale market. Even if they are of adequate size and can provide grid services on their own, their revenue might be trivial because of the limited capacity and flexibility. By subscribing to the FRS, either directly or through an aggregator or transactive market, the increased flexibility provided by all resources is expected to increase the revenue of the local DER aggregations from providing scalable transmission grid services.

For DERs subscribing directly to the FRS, their local DER controllers are required to follow the optimal DER dispatch signals provided by the FRS. They must also provide the information that is required by the FRS, as outlined in Section 5.3.1.2. In addition, resource flexibility is required information by the FRS from all subscribed resources. The flexibility defines the feasible operation bounds of a resource, and its format depends on the subscription option of the resource. Currently, not all local DER controllers have the capability to quantify such flexibility; therefore, examples of solutions to providing the required flexibility information will be developed in this project.

\subsubsection{Aggregators}

An aggregator is as an entity or agent that aggregates a group of assets or customers to act as a single participant in power system and/or power markets (Burger, et al. 2016) (Ikaheimo, Evens and Karkkainen 2010). Different DER aggregation projects have been proposed, launched, or implemented (Cook, et al. 2018). Compared with individual DERs that might be too small to participate in electricity markets, DER aggregation assembles a portfolio of DERs to meet the capacity requirement and thus can expand the economic gains for both the power system and the DER customers (Migden-Ostrander, et al. 2018). Some aggregators provide virtual power plant (VPP) services. Also, some aggregators use DERMS as their software platforms, where a DERMS is a software-based solution that provides, at a minimum, visibility of DERs through asset monitoring and DER scheduling and dispatch; and may also provide power and voltage control, resource valuation (U.S. Department of Energy 2015b), the ability to monitor and control DER aggregations and forecast DER capabilities (EPRI 2016a).

DERs can either be aggregated and controlled using a utility-DERMS, as discussed in Section 5.4.2.3, or through a third-party management system that is not under the DSO's control. We refer to such thirdparty management systems as "third-party DERMS." Multiple third-party DERMS providers exist, such as Siemens, Enbala, AutoGrid, and EnergyHub (Guidehouse insights 2020). Such DERMS typically manage smart inverters, combined heat and power units, and other distributed generation resources.

In addition to third-party DERMS, we consider that a variety of third-party aggregators possibly exist to manage multiple DERs, such as a fleet of EVs or a group of buildings. These third-party aggregators can either subscribe to the FRS and participate in activities to provide transmission grid services or directly bid in electricity markets on their own. If the third-party aggregator subscribes to the FRS, it is expected to submit the aggregated DER data, uncertainties, and forecasts to the FRS and follow the optimal schedules and control signals solved by the FRS. The operations of individual DERs are directly managed by the aggregator. If the third-party aggregator directly participates in the wholesale market, it will provide self-scheduled consumption/generation of resources under its direct control consistent with market gate closure in the day-ahead market. In this scenario, the third-party aggregator will be considered a "self-schedulable resource," as mentioned in Section 5.3.2.3. It should share its intended real and reactive operational schedules with the FRS prior to the day-ahead optimization. 


\subsubsection{Transactive Market Manager}

For FAST-DERMS, a key party in implementing DER controls in the transactive energy market is the TMM. The TMM is the "market maker," and its purpose is to form the market by which the parties can engage in price negotiation. The TMM will therefore serve as the pricing node that helps facilitate:

1. From the perspective of a consumer with DERs, recruit and reward load flexibility through transactive resources from both energy efficiency (e.g., reducing energy consumption in $\mathrm{kWh}$ ) and demand management (e.g., managing power in $\mathrm{kW}$ )

2. From the perspective of the DSO, meet the goal(s) of the utility, such as dispatching DERs to:

- Ensure, improve, or enhance the reliable delivery of power by the utility

- Manage, flatten, or control the variability of renewable generation or changing customer demand

- Better manage customer adoption and dispatch of rapidly changing technologies in connected homes and buildings (as a result of the increase of Internet of Things devices in buildings and building equipment)

- Help meet obligations to reduce fossil fuel usage through non-wires solutions (e.g., using the load side to realize change).

In an independent third-party configuration, for example, price could be directly seeded from feeder information by calculating the distribution locational marginal price to best serve the customer and distribution needs.

In this project, we are exploring two different TMM schemes defined by the direction in which price information is shared:

1. One-way communication: DERs receive and act given a price signal. The price formation will be influenced or modified by observation of the responses of DERs. In one-way communications approaches, DERs do not submit bids/offers; rather, their response is inferred from observation over time. Given the one-way communications mechanism, there are limitations on how quick and how "firm" the responses can be considered; therefore, it is expected that a combination of simulations, models, and historical data will be leveraged to characterize the uncertainty of responses

2. Two-way communications: DERs communicate their bids or offers (e.g., quantity of response or capacity at a given price) with the TMM. The bids/offers will directly influence the (next) price formation until settlement. The price formation will be influenced by the responses of DERs because DERs directly submit bids/offers.

\subsubsection{Communications}

Specific communications standards for different communications interfaces required by the FASTDERMS reference implementation, shown in Figure 6, are defined in the FAST-DERMS Communication System Design specification (Anandar 2021). These interfaces include device-level interfaces (1, 2, and $3)$, group-level control interfaces $(5,6$, and 7$)$, group-level transactive interfaces $(4,8$, and 9$)$ and transmission service interfaces (10). 


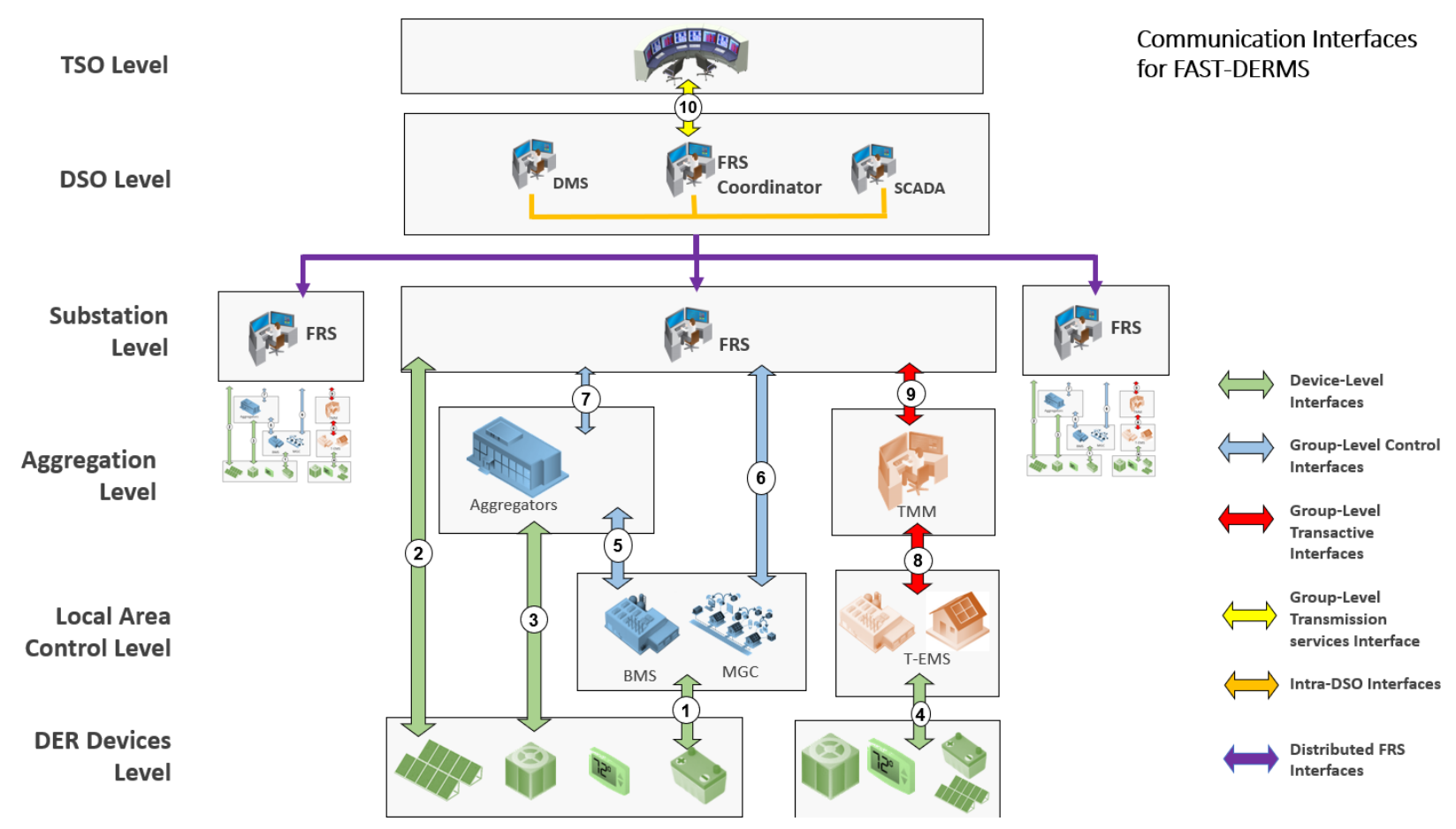

Figure 6. FAST-DERMS reference implementation communications interfaces

\subsection{Operational Concepts}

\subsubsection{Flexible Resource Scheduler}

The FRS determines the aggregate service quantities available for wholesale market bidding from all DERs connected behind a distribution substation. It operates on timescales consistent with both dayahead and real-time markets and provides control signals to the managed DERs, while ensuring distribution network constraints are respected. Using a stochastic optimization framework, the FRS is capable of managing individual DERs, aggregators of DERs, and DERs that participate based on realtime energy prices. The FRS coordinates with other distribution utility management systems, through the FRS Coordinator, to ensure it has updated distribution measurements and states, as well as constraints so that the aggregate TSO bids are solved based on distribution utility feedback.

\subsubsection{Control Method/Models}

A key function of the FRS will be to manage the uncertainty in the flexibility offers from distributed resources and thus provide a firm aggregate service to the TSO. This will be done by formulating an optimal power flow-based stochastic optimization using resources through a variety of subscription methods. The stochastic optimization will operate at two timescales:

- Ranging from 24-48 hours ahead for integration into day-ahead electricity markets 
- On a rolling basis every 5-30 minutes, ${ }^{1}$ with a time horizon of 2-4 hours. This instance operates as a model-predictive controller (MPC) that manages time-coupled resource states and integration into hour-ahead/real-time markets.

A separate dispatch algorithm will disaggregate TSO signals and provide real-time control signals to the various resources that have offered flexibility using the results of the MPC.

\subsubsection{Resource Models}

The model used in the optimization for the FRS will incorporate resource-specific and distribution network power flow constraints. In general, the FRS expects the following information from the resources $^{2}$ :

- Response model: This describes how the resources, or aggregations of resources, respond to the signal sent by the FRS. For transactive resources, this could be price-quantity curves; for aggregators or directly controlled resources, this could be simply following a reference signal. If a coupling exists between the resource's real and reactive power output, the coupling information would also be provided. If the resource is an aggregation, a model for how the aggregator will dispatch their resources could be included as well (e.g., proportional dispatch based on nameplate). This information is provided as part of the resource subscription.

- Feasible region (flexibility): Resources have constraints on both the time-dependent models of resource states (such as state of charge) and on the feasible operating regions of the resources (maximum power, reactive power, energy storage capacity, etc.). Feasible operating regions could be time dependent as well, such as in cases of demand response and EV aggregations. Providing the feasible region in which a resource's states can be operated represents the flexibility of the resource and can be provided on a continuous basis because it could evolve with time.

- Uncertainty: Each resource will have uncertainty associated with forecasted flexibility that will also be used by the FRS. A probability density function for each point estimate is ideal, though the exact model of uncertainty to be provided is open for discussion.

- Locational information: Resources will need to provide locational information, ideally which bus and phase(s) in the network model they connect to. If they belong to an aggregation that is within an area of the network, they can provide the aggregation information instead of a specific bus. This information will be used by the FRS to ensure that network constraints are satisfied. We can assume that the DSO can supply the network location based on the resource's service address and its subscription method.

- Cost information (if transactive): Although the resource will specify during subscription that it will be responsive based on price, the shape of that response might change over time. A transactive resource must provide its cost of generation/price response as a time series of monotonic price-quantity curves. It is anticipated that price-responsive resources will have varying abilities to provide such types of information. The TMM, another component in the FAST-DERMS architecture, will be capable of developing/refining price response models where resources are unfit to provide them.

It is expected that this information will be in different forms for different resource types. Also, if a resource is involved in multiple services or services at multiple timescales, it might need to provide

\footnotetext{
${ }^{1}$ This time is still under investigation and will be finalized in the next version of the document. In general, it could be $5,15,30$, or 60 minutes, depending on the complexity of the model and the needs of the architectural design.

${ }^{2}$ Note that to the FRS, a resource could be a single resource or an aggregation. The information presented applies to both unless explicitly stated.
} 
information for each service. As of now, we intend to model up to three different types of flexible resources: load, generation, and storage. Another option could be to create a single generalized DER model that can be used to represent all three types in the FRS. Aggregations of resources will be modeled as a single resource, which might make the generalized model the best option.

\subsubsection{Addressing Computational Tractability}

We anticipate that making the stochastic optimization problems tractable will require network-reduction techniques to reduce computational complexity. This is an opportunity for other DERMS/ADMS functions to supply meaningful information to the FRS. ADMS and third-party DERMS applications could identify critical infrastructure locations (buses or lines) or provide live network parameter updates to ensure that specific buses are maintained in the reduced-form optimization that would improve the performance of the FRS. Other approaches to reduce the computational burden will be examined as well, such as careful choice and weighting of scenarios if scenario-based optimization is a selected solution methodology. Further, the day-ahead problem might require a different solution methodology and/or formulation than the near-real-time MPC.

\subsubsection{Forecasted Input Requirements}

Creating day-ahead offers for the distribution network as a whole requires the use of forecasts for the remainder of the load in the network in addition to what controllable resources are providing the system. Probabilistic nodal load forecasts will be created on timescales consistent with the day-ahead and hourahead controllers. Similarly, there will also be probabilistic forecasts of insolation and/or PV production for the DERs directly managed by the FRS.

\subsubsection{Registration and Subscription Methods}

During the registration process, DERs in the distribution network that have opted to participate in grid service provision will need to effectively "subscribe" to an agreement with the DSO. Agreements for resource integration specify the type of incentive that a resource will receive and respond to and the level of aggregation of the resource. Appendix B lists some existing settlement plans used by utilities to recruit DERs to participate in demand response programs and/or to provide grid services.

Based on the incentive choice, sources are categorized into two main groups: (1) transactive resources whose consumption is dependent on the electricity price and (2) contractually obligated resources that are under a prior contract to offer flexibility and are under direct control of the FRS. The aggregation level specifies whether a resource is an individual DER or an aggregation of multiple resources and whether those resources are co-located - and if not, how much information the FRS has about the location of resources. The combination of incentive choices and aggregation level defines the way in which a resource is modeled and controlled in the FRS.

FAST-DERMS does not limit the number of grid services that DERs can participate in. DERs can choose the same or different subscription packages for different services at will-e.g., DERs can subscribe as a transactive resource for energy market participation and subscribe as a contractually obligated resource that is under the direct control of the FRS for ancillary services in the transmission grid; therefore, DERs can receive benefits from different programs for providing different services. But DERs are not allowed to subscribe through different subscription methods for the same service to avoid "double counting." Under current regulations, double counting-i.e., receiving benefits from different programs for the same service - is prohibited; thus, by limiting DERs to one subscription method per service, FAST-DERMS can eliminate the possibility of double counting when scheduling the DERs. 
Resources in the distribution network can also opt out of participation in the FRS by declaring themselves to be self-scheduled. These resources could opt for direct participation in wholesale markets or other outof-market activities that require distribution access. But these resources must interact with the FRS to provide operation schedules because their production/consumption impacts the substation meter where TSO services are measured.

\subsubsection{Incentive Options}

Incentive options govern the method of control by which a resource participates in the FRS - based either on price or on direct power set point. The two main categories of incentive structures for resources subscribing to the FRS are transactive and capacity obligated. Transactive resources receive remuneration primarily based on a savings they can achieve by aligning their consumption with a real-time electricity price signal. Contractually obligated resources receive remuneration from participation primarily from an existing capacity contract with their DSO. The flexible portion of their capacity will be under direct control of the FRS, responding to real and/or reactive power set point commands.

From the perspective of the FRS, all transactive resources will interact with the FRS in the same manner. They will provide hourly price-quantity curves or "bids" for energy that are aligned with decision-making for day-ahead market interaction. They will have a recourse opportunity to update that information before gate closure for real-time markets. They will provide a measure of uncertainty on the output of those curves. Additionally, they will receive hourly pricing that results from TSO day-ahead market clearing (for planning purposes) and 5-minute energy dispatch prices that will be settled in real time. We anticipate that most resources cannot provide the full suite of data needed for the probabilistic optimization of transactive resources, so FAST-DERMS includes a TMM that will provide aggregation and integration solutions for these resources. The TMM will act as an aggregator for most transactive services, maintaining the single communications and data structure required by the FRS. The ways in which a resource might interact with the TMM are described in Section 5.3.3.

Contractually obligated resources are those that the DSO has previously contracted to provide flexibility. These resources are dispatched directly with a real and/or reactive power command from the FRS realtime control. They provide the feasible real and reactive power bounds around which they might be controlled as well as schedules of any baseline power being consumed or produced under the absence of a service request. Where a form of energy storage exists, they might provide their initial state of charge and any off-of-meter changes in energy storage throughout the day (e.g., EVs disconnecting from charging infrastructure in a parking garage). We further categorize contractually obligated resources as:

1. Resources that are under a capacity obligation with a predefined contract to provide flexibility. If valuable, day-ahead and MPC optimizations can provide these resources with their expectation of power set points based on the optimization runs, though the ability to respond to the real-time dispatch controller would be more valuable. It is anticipated that these resources would communicate their flexibility and associated uncertainty to the FRS for integration into both the day-ahead and realtime optimizations. Contractually obligated resources could also include energy storage limits in their resource definition.

2. Resources that are under a capacity obligation with a predefined contract to provide flexibility with dispatch limitations. These resources are very similar to those described above, but they face dispatch limitation seasonally or over another horizon greater than that of the FRS. This is meant to be similar to legacy demand response programs in which a limited number of calls is allowed by the program per season. The FRS would include an explicit risk of running out of these resources in the objective value, though the approach would be based largely on engineering intuition because explicitly quantifying this risk is out of the project's scope. 
We acknowledge that under certain circumstances (e.g., in advance of public safety power shutoff events), the capacity obligation constraints and restrictions might be revised to follow the regulations of governing authorities. From the perspective of the FRS, the resource models will be general enough to accommodate such scenarios.

\subsubsection{Aggregation Options}

There are five possible levels of aggregation:

1. Individual metered resources. This is a single resource located at a specific bus on the network model, and its interaction is measured at that meter. From the perspective of the FRS, there is not a difference between communicating directly with the resource and communicating with a third-party aggregator that represents them and relays the dispatch signal.

2. Aggregations of co-located resources behind a single meter. This resource could comprise multiple resource types and exists at a known network location. The expectation is that a local resource manager, such as a BMS, would be responsible for disaggregating commands/price response. Some examples of these resource types include prosumer commercial buildings with multiple DERs, a commercial EV fleet parking lot, or a microgrid.

3. Aggregations behind multiple meters-resources known. In the process of registering their aggregation, the aggregator indicates the locations and capacities of all their resources in the network; however, the resource the FRS uses is the whole aggregation, and it integrates only with the aggregator; thus, when the FRS sends a dispatch command to the aggregator, the locations that the aggregator uses to respond to that dispatch are unknown. The FRS must represent this dispatch with some model of the spatial uncertainty of the dispatch.

4. Aggregations behind multiple meters-resources partially known. The aggregator indicates to the FRS the total capacities of its aggregation (and associated uncertainties) in accordance with its bidding process timeline. The FRS has a general knowledge of where and what resources are in the network (such as that they are inside a particular "zone" of the distribution network). Integration is purely with the aggregator.

5. Aggregations behind multiple meters-resources unknown. The aggregator indicates to the FRS the total capacities of its aggregation (and associated uncertainties) in accordance with its bidding process timeline. The FRS has no knowledge of where or what resources are in the network (though perhaps there could be boundaries such as a group of laterals). Integration is purely with the aggregator. This carries the most uncertainty of any aggregation model and would be the least valuable, though likely much easier to assemble.

\subsubsection{Non-Subscribing Resources}

These are also resources that will not subscribe to and provide their flexibility to the FRS. Some resources could participate directly in the wholesale market. We assume that these resources will have a wholesale distribution access tariff ${ }^{1}$ in place, and thus these resources will require interaction with the DSO to gain access. From the perspective of the FRS, these resources become self-scheduled resources, and they are treated as inflexible net load on the system. Although these resources are not providing their flexibility to the FRS, they are located inside the distribution network that is managed by the FRS; therefore, these self-schedulable resources will be required to provide their intended power/energy operational schedules prior to the day-ahead optimization. If they provide economic bid curves to the wholesale market, these

\footnotetext{
${ }^{1}$ Today, a distribution-connected resource needs a contract with the utility to access wholesale markets. This is called a Wholesale Distribution Access Tariff.
} 
could be provided in lieu of scheduled operation. We also assume that the wholesale distribution access tariff would have provisions to revoke access or to penalize the resource if it significantly deviates from its schedule. This scheme will ensure that the FRS can assume that the information is relatively accurate and perform its network optimization.

Some nonutility-owned resources could be operated or managed by themselves and do not participate in the wholesale market. Because they do not directly interact with the FRS/DSO, we assume that the FRS has no knowledge of these DERs other than their locations and capacities if they are registered with the distribution utility. These resources are both uncontrollable and introduce high uncertainty into the FRS optimization models.

In practice, these non-subscribing resources need to go through an interconnection procedure before being connected to the distribution grid; thus, their capacities and other basic parameters are registered, and they are considered to be available to the FRS. Although the FRS does not have the authority to directly dispatch these resources, the registration information will be used by the FRS for uncertainty modeling and optimization.

\subsubsection{Transactive Market}

In this project, we are exploring two different TMM schemes defined by the direction in which price information is shared:

1. One-way communication: DERs receive and act given a price signal. The price formation will be influenced or modified by observation of the responses of DERs. In one-way communication approaches, DERs do not submit bids/offers; rather, their response is inferred from observation over time. Given the one-way communication mechanism, there are limitations on how quick and how "firm" the responses can be considered; therefore, it is expected that a combination of simulations, models, and historical data will be leveraged to characterize the uncertainty of responses.

2. Two-way communication: DERs communicate their bids or offers (e.g., quantity of response or capacity at a given price) with the TMM. The bids/offers will directly influence the (next) price formation until settlement. The price formation will be influenced by the responses of DERs because DERs directly submit bids/offers.

\subsubsection{One-Way Communication Scheme}

The one-way communication scheme results from a consideration of how to best organize technology in buildings and considering the building/grid relationship. It is also significantly inspired by appropriate lessons from the success of information technology by considering that the amount of diverse legacy DER technologies we have today will be dwarfed by what we will have in 10 years. The one-way communication scheme helps address "backward compatibility" as grid technologies evolve.

Energy and grid services could be separated into three buckets: (1) those concerned primarily with the overall balance of the supply and demand of energy to minimize costs, (2) those concerned with power quality and related energy topics, and (3) those that address system capacity constraints that are primarily local. Further, the use of direct load control, event-based demand response, and aggregators began at a time when DER control was a priori. Through their existence, however, these schemes have slowly worked on pricing, response, and "firmness" a posteriori. For these reasons, one-way communication schemes are important to revisit. 
In the one-way communication diagram shown in Figure 7, a price signal is passed from the FRS to the transactive resources, where the DERs operate themselves and "consume" energy. A response evaluator is introduced in the TMM to observe the "consumed" energy (e.g., the observed feeder-level energy) or response to the provided price. The response evaluator will send the estimated response as a function of the price change that was observed to the FRS. As explained, the responses of the DERs can be estimated by the response evaluator based on historical data, demand elasticity, etc.

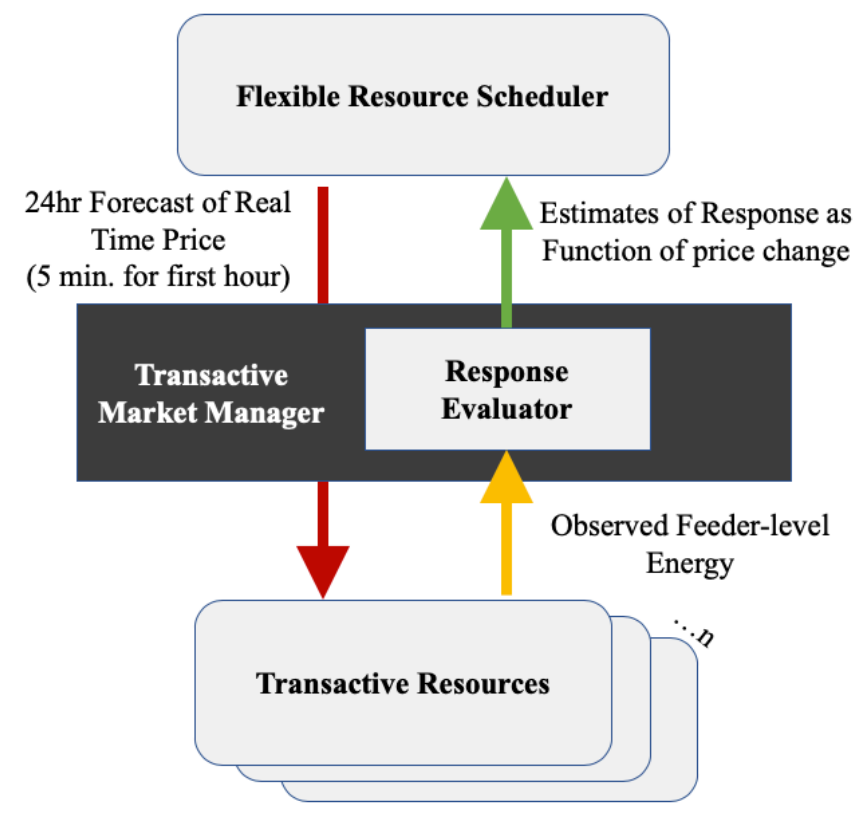

Figure 7. Transactive energy market based on one-way communication

\subsubsection{Two-Way Communications Scheme}

In the two-way communications scheme, shown in Figure 8, the price and response can be communicated bidirectionally between the parties. The TMM serves as the intermediary between the transactive resources and the FRS. The two-way communications scheme consists of the following steps:

- The FRS sends a forecast as a high-/low-price band derived from its needs to the TMM.

- The TMM calculates a flexibility band (as a bid price) and sends the forecast to the transactive resources.

- The transactive resources optimize their energy schedules with respect to the received price forecast and generate the corresponding quantity of responses that are sent back to the TMM.

- The TMM evaluates the received quantity offers at the bid prices from the transactive resources and settles the local market according to the pricing scheme with respect to distribution system constraints, or it engages in price discovery/negotiation again (repeating steps 2 and 3 ) to arrive at a settled price and quantity.

The settled DER responses will then be sent to the FRS. The TMM can augment the data to include an estimation of uncertainty to characterize the anticipated firmness of the DERs. 


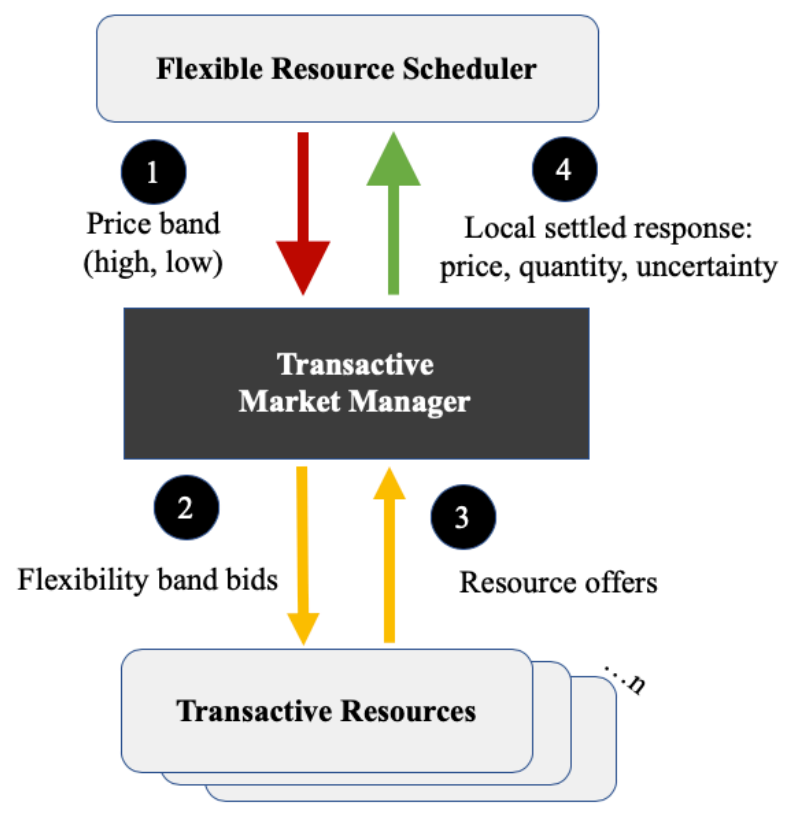

Figure 8. Transactive energy market based on two-way communications

\subsubsection{Secure Grid Operations}

To secure grid operations with the adoption of FAST-DERMS, this solution incorporates a two-step process to enhance the security of FAST-DERMS. As depicted in Figure 9, the first step is to detect anomalies using a hybrid method incorporating both DSSE and a machine learning algorithm. The hybrid anomaly detection method can effectively defend against false data injection attacks targeted at both system model and real-time DER measurements. Once an anomaly is detected, the FRS will be informed for re-optimization, and local volt/VAR responses of inverter-based DERs will adaptively stabilize the network voltage and frequency (xx). Through such a two-step procedure, FAST-DERMS can help secure grid operations in the presence of unexpected events to some extent. We recognize that passive anomaly detection has limited capability in handling sudden, large-scale failures, and a holistic approach that can prepare for, detect, mitigate and endure attacks is needed to comprehensively secure grid operations. 


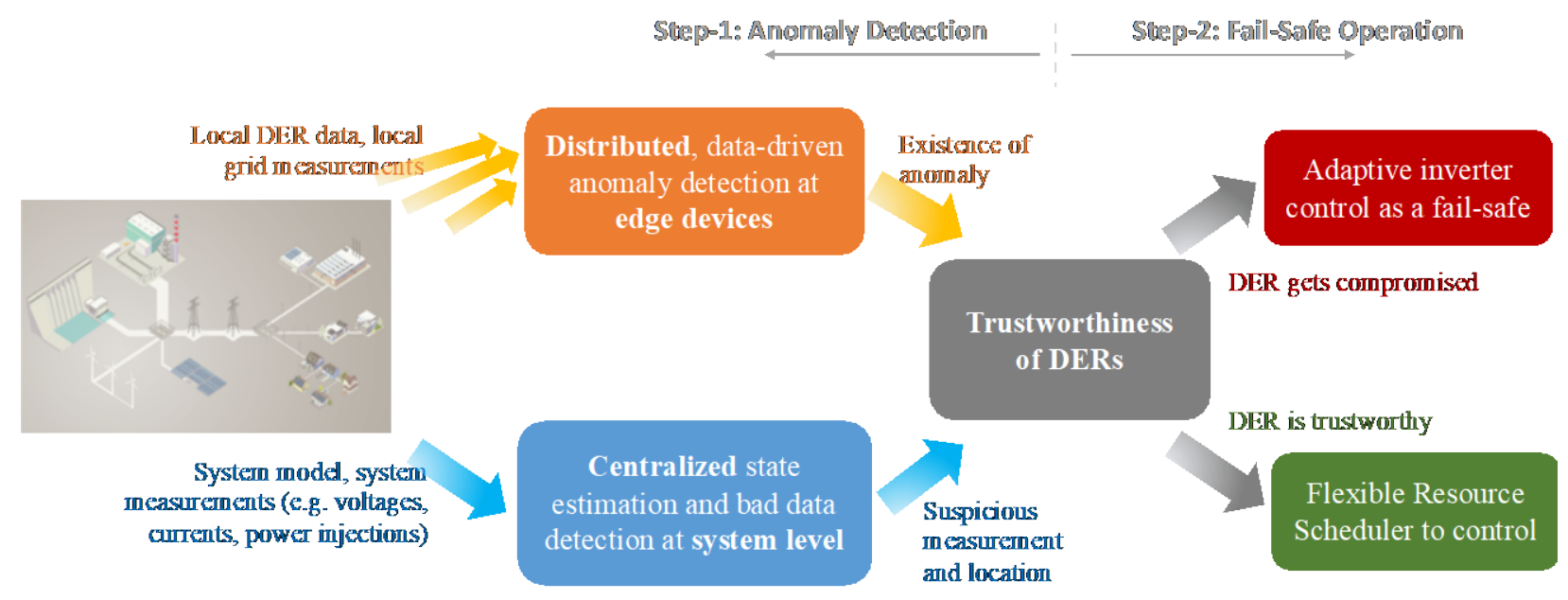

Figure 9. A two-step process adopted in the FAST-DERMS reference implementation to secure grid operations

\subsection{Interaction Patterns}

\subsubsection{Interaction with Transmission System Operator}

In this section, we assume that the TSO is an ISO or an RTO that operates wholesale markets through competitive market mechanisms. In Section 5.4.4, we discuss the operation of the FRS in traditionally regulated markets.

FAST-DERMS is following the total DSO approach, as introduced in Section 3.7-i.e., the DER aggregators operating resources in a distribution system should go through the DSO to participate into the wholesale market.

According to market regulations, the FRS Coordinator might not be eligible to directly bid in the ISO market. For example, the California Independent System Operator (CAISO) requires all businesses except for the acquisition and holding of congestion revenue rights to be conducted through approved and registered entities called "scheduling coordinators." For simplicity, hereafter we use the term FRS to refer jointly to the individual FRSs and to the FRS Coordinator.

The primary responsibilities of scheduling coordinators are to:

- Represent generators, load-serving entities, proxy demand resources, reliability demand response resources, importers, and exporters

- Provide North American Electric Reliability Corporation (NERC) tagging data

- Submit bids and inter-scheduling coordinator trades

- Settle all services and inter-scheduling coordinator trades related to the CAISO markets

- Ensure compliance with CAISO tariff

- Submit annual, weekly, and daily forecasts of demand.

Note: The FRS is not a scheduling coordinator, and it does not necessarily make the DSO become a scheduling coordinator. Two scenarios could exist: 
1. The DSO is not a certified scheduling coordinator. In this scenario, the FRS generates energy bids and submits bidding information to its corresponding scheduling coordinator for market clearing.

2. The DSO is a certified scheduling coordinator. In certain cases where the DSO can act as a scheduling coordinator, the FRS can be configured to directly submit its bids to the wholesale market on the DSO's behalf.

In this project, we consider the scenario where the DSO is not a scheduling coordinator. Figure 10 illustrates the responsibilities of the FRS and the scheduling coordinator along the market operation timeline. As shown in Figure 10, there is a clear boundary between the responsibilities of the FRS and the scheduling coordinator. The FRS manages the market participation strategies of DERs and submits bids to the scheduling coordinator on behalf of the DSO. The scheduling coordinator aggregates bid information and directly interacts with the market operator. When the market is cleared, the scheduling coordinator allocates the market settlement to the corresponding FRS. The FRS then takes the responsibility to disaggregate the settlements and notify its subscribers about their final energy schedules.

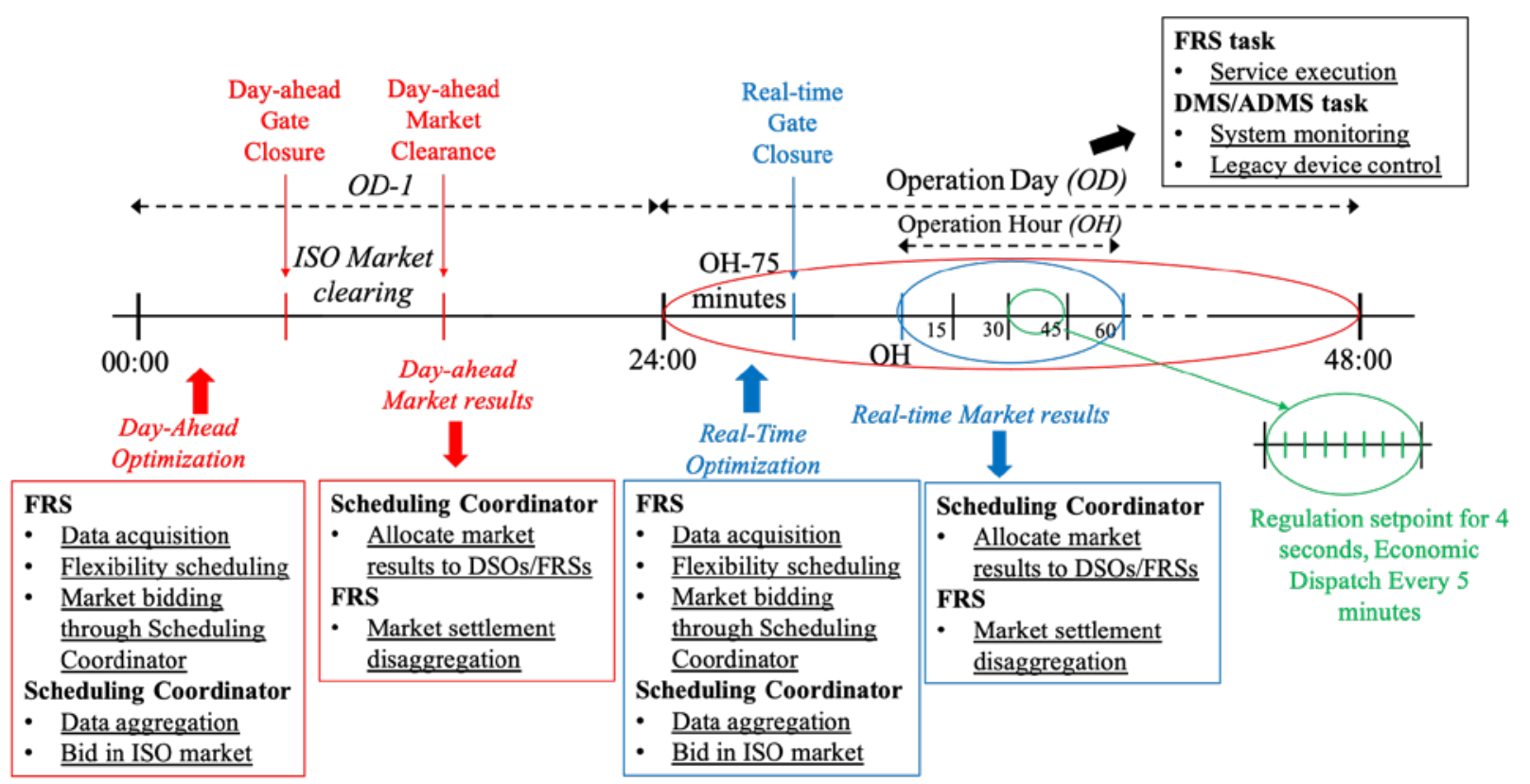

Figure 10. Responsibilities of the FRS and scheduling coordinator based on the market timeline

\subsubsection{Interactions with Existing Distribution Utility Systems}

FAST-DERMS focuses on the control integration of DERs through the FRS. Legacy devices-such as load tap changers, voltage regulators, capacitor banks, and line switches — are still managed and monitored by existing utility management systems.

As described in Section 5.2.2, the FRS provides two major functions:

1. Provide a bid or schedule to a wholesale market on behalf of the DSO as well as bids or schedules of any capacity-based ancillary services and other nonmarket services.

2. Disaggregate grid control signals in real time to the various resources subscribed to the FRS (as described in Section 5.3.2) to meet the objectives for the entire network. 
In addition, the FRS needs to coordinate with existing distribution utility management systems to satisfy both distribution voltage and thermal constraints and to maintain distribution system reliability.

The FRS requires a certain amount of system information that can be acquired directly from SCADA and geographic information system (GIS) if utilities do not have a DMS or an ADMS. For utilities that do have a DMS or an ADMS, the FRS can be embedded in the existing DMS or ADMS platforms, or it can acquire system information from the DMS/ADMS to perform its task. This section provides an overview of the relationship between the FRS and existing utility systems

\subsubsection{Supervisory Control and Data Acquisition}

For DSOs that do not have a DMS or an ADMS, the FRS requests system topology information, DER locational information, and real-time feeder head measurements (e.g., active and reactive powers, voltages, currents) from the SCADA system. In such systems, the legacy devices are typically operating under local control. In addition, it is important for the FRS to consider the local capacitor operations and regulator tap position changes when solving its optimization problems.

\subsubsection{Distribution Management System and Advanced Distribution Management System}

For DSOs that have a DMS or an ADMS, the FRS can be embedded in the existing DMS or ADMS platform, or the FRS can acquire system information from the DMS/ADMS. In either configuration, the FRS performs best when operating in a total DSO model, where all resources located in the network are either responsive to the control and/or price signals or provide self-schedules on time to limit exposure to poor market and control performance measured at the substation meter. There are a few opportunities to leverage the capabilities of a DMS/ADMS in FAST-DERMS:

1. The DMS/ADMS updates topology as switching decisions are made and provides set point schedules for legacy devices (e.g., capacitor banks and voltage regulators). Ideally, this is done prior to gate closure in day-ahead wholesale markets because significant topology changes can have large impacts on resource availability for the distribution feeder aggregation.

2. Network reduction will be used in the FRS to reduce optimization computational complexity. There could be some critical buses that should not be merged or simplified in the network reduction, and these bus locations should be provided by the DMS/ADMS to the FRS.

3. The DMS/ADMS checks the feasibility of the power flow resulting from the FRS to satisfy local distribution system operational requirements with the potential control actions on legacy devices and flags any buses that could result in a constraint violation, prompting an update of constraints and rerunning the FRS to achieve feasibility. This feasibility check will be useful as a safety check, and it will be valuable to ensure reliable system operation given the simplifications and assumptions made to perform multiperiod optimal power flow in the FRS.

If a distribution utility does not have a DMS or an ADMS, the FRS will operate as a stand-alone system, and it can run full-system power flow to perform a feasibility check.

\subsubsection{Distributed Energy Resource Management System}

As a complement to a DMS that controls only legacy grid assets, some DSOs also have a DERMS or an ADMS providing DERMS functions to control utility-owned DERs, such as solar power plants and utility-scale BESSs. DERMS applies to software that can integrate the needs of utility grid operations 
with the capabilities of flexible demand-side energy resources at the edges of the grid (Greentech Media 2017) and manage diverse and dispersed DERs, both individually and in aggregate, to support multiple objectives related to distribution grid operations, end-customer value, or market participation. We call such systems "utility-DERMS." For such utilities, the FRS is a complement to the existing utilityDERMS, and it will add extensive energy scheduling capability for both privately owned and BTM DERs. The coordination between the FRS and utility-DERMS varies significantly depending on whether the FRS can schedule the DERs operated by the utility-DERMS or not.

If the FRS can schedule these DERs, the FRS will acquire DER data from utility-DERMS and treat them as a group of flexible resources managed by a utility-owned aggregator. The utility-DERMS will control its DERs to follow the schedules optimized by the FRS.

If the FRS cannot schedule these DERs, the utility-DERMS will schedule its DERs in accordance with its original rules, and the FRS might receive the schedules of utility-owned DERs and treat them as known, non-dispatchable loads.

\subsubsection{Interactions with a Wholesale Market Operated by a Transmission System Operator}

This section provides an example of an FRS interacting — through a scheduling coordinator — with a wholesale market operated by a TSO for a distribution utility that has an ADMS deployed. As before, we use the term FRS to refer jointly to individual FRSs and to the FRS Coordinator. A sequence diagram in Figure 11 shows the process flow of the FRS participating in the wholesale market and the interactions between the FRS and the other relevant entities. 


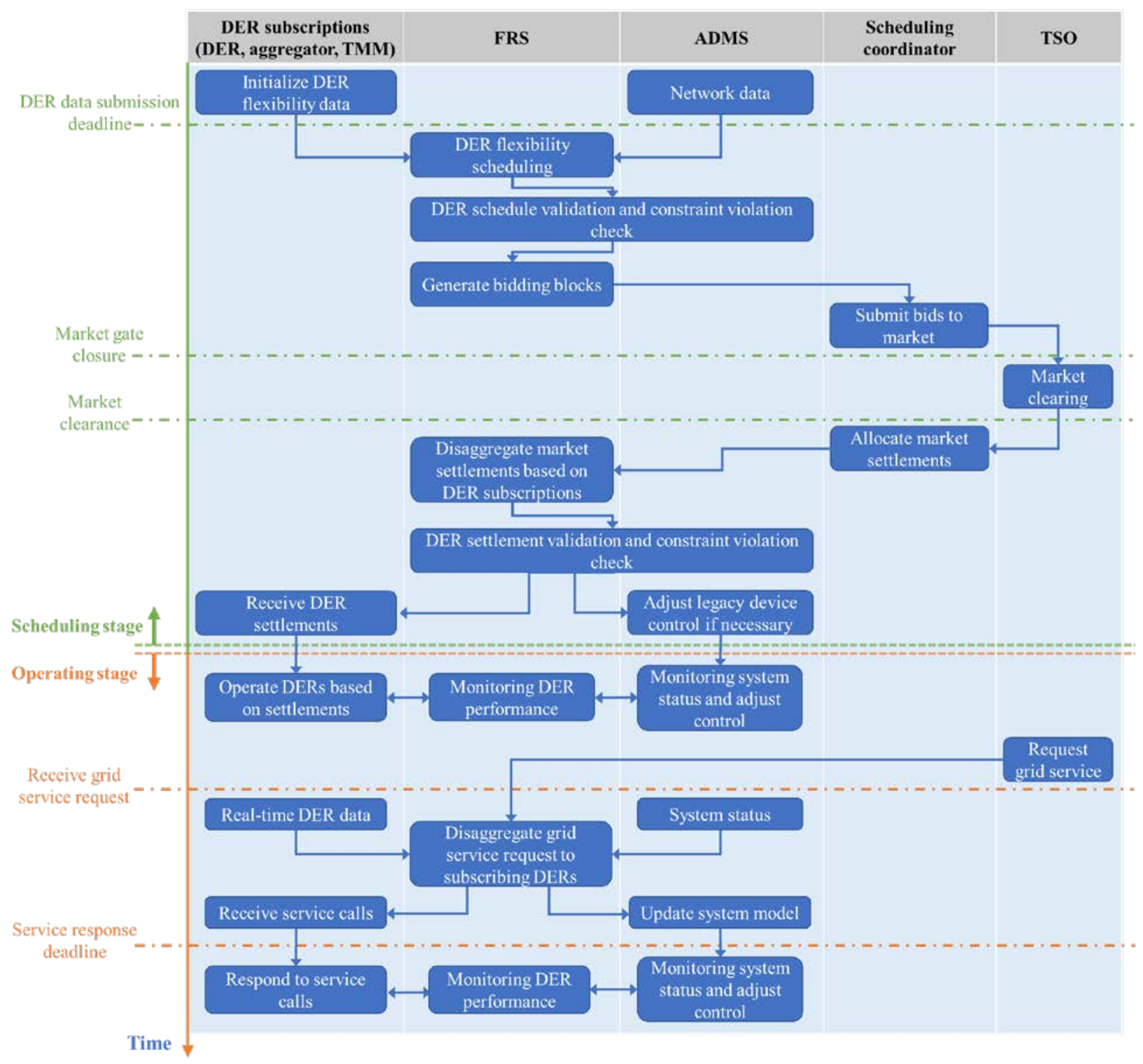

Figure 11. FRS process flow to participate in wholesale markets

The interactions are divided into two stages: the scheduling stage and the operation stage. An explanation of these two stages is provided in the following.

\section{(1) Scheduling Stage}

In the scheduling stage, the FRS has three major roles:

- Optimizing DER schedules

- Coordinating with the ADMS to avoid distribution operation constraint violations

- Bidding into electricity markets.

To optimize DER schedules, the FRS needs to retrieve the following data before scheduling DERs:

- Network topology and parameters as well as the settings of legacy devices from the ADMS 
- Resource model information, as discussed in Section 2.2.1.1, from the resources that have subscribed to the FRS

- Intended power/energy operational schedules from the self-scheduled, nonparticipating resources

- Locations and capacities of other nonparticipating DERs (not self-scheduled), if known, from the DSO

- Historical operating data (market price, etc.) from the FRS's own database.

To coordinate with the ADMS:

- The FRS sends its DER schedules to the ADMS.

- The ADMS runs full-network power flow and performs a feasibility check based on the DER schedules submitted by the FRS, load forecast, and legacy device status. If distribution network constraints are violated, the ADMS will notify the FRS, and the FRS will need to revise the DER schedules to avoid the violations.

To participate in electricity markets:

- The FRS generates bidding blocks (quantity and price) based on the aggregated resources' flexibility for different grid services and submits the net load bidding blocks to the scheduling coordinator for market clearing.

- The FRS receives cleared market settlements from the scheduling coordinator.

- The FRS allocates disaggregated awards and settled prices to its subscribed resources for day-ahead and intra-hour planning.

\section{(2) Operating Stage}

In the operating stage, the major role of the FRS is managing the responses of subscribed resources to the grid service requests. The FRS might receive grid service requests throughout the operation period. Based on the service request and the scheduling solution determined during the scheduling stage, the FRS will determine the disaggregated real-time control signals and send them to its subscribed resources. The actual responses of the subscribed resources will be collected and monitored by the FRS. If a grid service request cannot be fulfilled by some resources that have subscribed to those services, the FRS will perform a real-time redispatch to meet the grid service target through the control of other available resources.

Because of the fast response requirement of real-time operation, unlike the scheduling stage, we assume that there is no interaction for a feasibility check and decision revision between the ADMS and the FRS. Instead, it is expected that the ADMS can provide the FRS with the operation/control laws of its controlled legacy devices or the acceptable range of the power injection at the bus locations of the FRS resources so that the real-time control signals generated by the FRS will not violate distribution network operation constraints (such as voltage or thermal limits). But it is possible that some ADMS platforms cannot provide such information; in that case, alternative solutions will need to be found to address the constraint violation issues. 


\subsubsection{Interactions within a Traditionally Regulated Market Environment}

Utilities in traditionally regulated markets ${ }^{1}$ are frequently vertically integrated, owning generation, transmission, and distribution (Coley Girouard 2015). In a vertically integrated utility, the FRS still aggregates and optimizes the operations of all DERs located downstream of a substation in a physical distribution network and provides a schedule for the network's net load (and in rare instances, net generation) to the utility's transmission operator on behalf of the distribution operator as well as bids or schedules for any capacity-based ancillary services while maintaining voltage and thermal constraints in the distribution system.

So far, we have considered the FRS scheduling DER flexibility in competitive markets operated by an ISO or RTO. Although competitive markets are absent in a traditionally regulated market, this does not introduce unique technical difficulties for deploying flexible DERs to support transmission-level operations. The FRS can still be relied on for aggregating and optimizing DERs; thus, this subsection briefly reviews the interaction between the FRS and other entities within a traditionally regulated market environment.

A general conceptual diagram of FAST-DERMS when adopted by utilities in a traditionally regulated market environment is shown in Figure 12. The key difference between Figure 12 and Figure 5 lies in the interaction of the FRS with the transmission system. Because vertically integrated utilities in a traditionally regulated market environment do not rely on a competitive electricity market to schedule generating resources, the FRS will not communicate with a scheduling coordinator or market operator. Instead, the FRS still needs to solve its optimization problem but directly submits the schedule for the network's net load (and in rare instances, net generation) to the transmission operator in the utility company for its scheduling and dispatch purposes. The FRS subscription methods described in Section 5.3.2 are still applicable for DERs in a traditionally regulated market environment.

\footnotetext{
${ }^{1}$ As defined in the Federal Energy Regulatory Commission's Energy Primer: A Handbook of Energy Market Basics, June 2020, available at https://www.ferc.gov/sites/default/files/2020-05/energy-primer.pdf.
} 


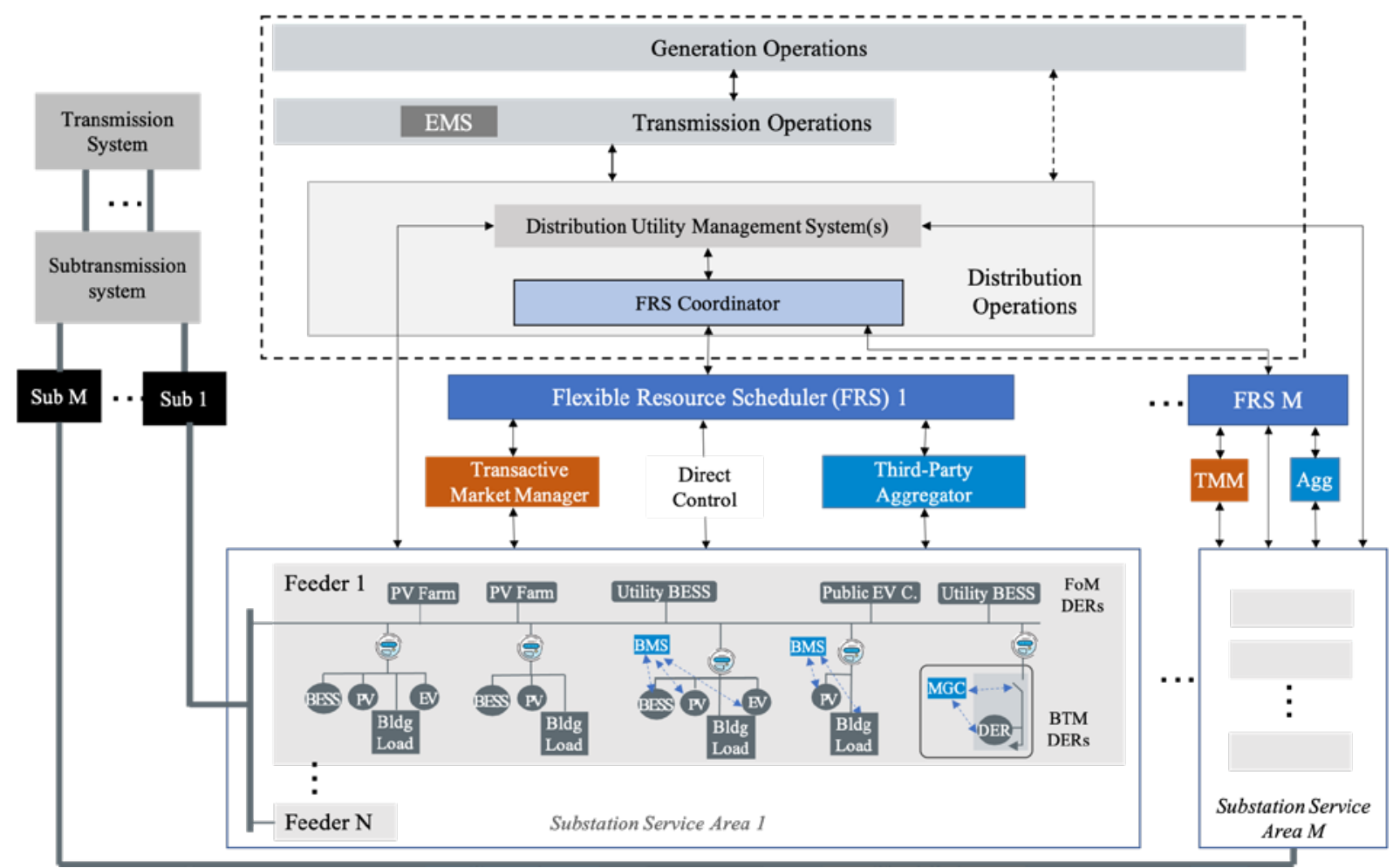

Figure 12. Conceptual diagram of FAST-DERMS applied to a vertically integrated utility in a traditionally regulated market environment

At present, although a utility company is vertically integrated, the operations of the transmission system and the distribution system might still be completely isolated. Also, there exist some vertically integrated utilities in which DERs are owned and operated by the generation side, which oversees the operations of generators, but the generation operators do not have connections with distribution operators. To reflect such scenarios, we use dashed lines in Figure 12 to denote the connections between the generation operations and the distribution operations. With the increasing integration of DERs, to fully exploit the value of DERs, we envision that both communications and coordination will exist among generation, transmission, and distribution operations within vertically integrated utilities in a traditionally regulated market environment in the near future. 


\section{References}

ABB. 2019. "DERMS looking ahead." https://library.e.abb.com/public/8c470712bab44130b06b3d3da74c7bcf/DERMSLooking-Ahead 9AKK107492A2126-US.pdf.

Abrishambaf, Omid, Fernando Lezama, Pedro Faria, and Zita Vale. 2019. "Towards transactive energy systems: an analysis on current trends." Energy Strategy Reviews 100418.

Agalgaonkar, Yashodhan P., and Donald J. Hammerstrom. 2017. "Evaluation of smart grid technologies employed for system reliability improvement: Pacific Northwest smart grid demonstration experience." IEEE Power and Energy Technology Systems Journal 24-31.

American Electric Power. 2011. "Direct load control event." https://smartgrid.epri.com/UseCases/Direct\%20Load\%20Control\%20Event ph2add.pdf.

Anandar, Jithendar. 2021. "FAST-DERMS Communication System Design Specification". Electric Power Research Institute.

Ardani, Kristen, Eric O'Shaughnessy, and Paul Schwade. 2018. "Coordinating distributed energy resources for grid services: a case study of Pacific Gas and Electric". Golden, CO: NREL.

Argonne National Laboratory. 2017. "Foundational report series: advanced distribution management systems for grid modernization."

Black \& Veatch Management Consulting, LLC. 2020. "Distribution System Operator (DSO) Models for Utility Stakeholders: Organizational Models for a Digital, Distributed Modern Grid." https://www.bv.com/sites/default/files/202001/20\%20Distribution\%20System\%20Operator\%20Models\%20for\%20Utility $\% 20$ Stakeh olders $\% 20 \mathrm{WEB} \% 20 \% 281 \% 29$.pdf.

Burger, Scott, Jose Pablo Chaves-Avila, Carlos Batlle, and Ignacio J. Perez-Arriaga. 2016. "The value of aggregators in electricity systems." http://energy.mit.edu/wpcontent/uploads/2016/01/CEEPR WP 2016-001.pdf.

California Energy Commission \& California Public Utilities Commission. 2015. "Recommendations for utility communications with distributed energy resources (DER) systems with smart inverters, Smart Inverter Working Group Phase 2 Recommendations, Draft v9." https://www.energy.ca.gov/sites/default/files/201905/SIWG Phase 2 Communications Recommendations for CPUC.pdf.

California ISO. 2020a. "Business practice manual for market operations." Mar. https://bpmcm.caiso.com/Pages/BPMDetails.aspx?BPM=Market\%200perations.

—. 2015a. "Energy storage and distributed energy resources (ESDER) stakeholder initiative." Jul.

http://www.caiso.com/InitiativeDocuments/EnergyStorageandDistributedEnergyResourc es-IssuePaperStrawProposal.pdf.

—. 2015b. "Flexible ramping product: revised draft final proposal." Dec. http://www.caiso.com/Documents/RevisedDraftFinalProposal-FlexibleRampingProduct2015.pdf.

—. 2016. "Frequency response phase 2." Dec. https://www.caiso.com/Documents/IssuePaper_FrequencyResponsePhase2.pdf.

—. 2020b. "PDR-DERP-NGR summary comparison matrix." http://www.caiso.com/Documents/ParticipationComparison-ProxyDemandDistributedEnergy-Storage.pdf.

California Public Utilities Commission. 2020a. Distributed energy resources. https://www.publicadvocates.cpuc.ca.gov/der.aspx. 
-. 2020b. Rule 21 Interconnection. https://www.cpuc.ca.gov/Rule21/.

Cleveland, Frances, and Annabelle Lee. 2013. "Cyber security for DER systems." EPRI. Jul. https://smartgrid.epri.com/doc/der\%20rpt\%2007-30-13.pdf.

Coley Girouard, and Danny Waggoner. 2015. How much do you know about your electric utility. Feburary 17. https://blog.aee.net/how-much-do-you-know-about-your-electric-utility.

Cook, Jeffrey J., Kristen Ardani, Eric O'Shaughnessy, Brittany Smith, and Robert Margolis. 2018. Expanding PV value: lessons learned from utility-led distributed energy resources aggregation in the United States. Golden, CO: NREL.

Department of Energy Federal Energy Regulatory Commission. 2020. "Participation of Distributed Energy Resource Aggregations in Markets Operated by Regional Transmission Organizations and Independent System Operators."

ENTSO-E. 2020. Transmission system operator. http://www.entsoeevent.eu/transmission_system_operator.html.

EPRI. 2016b. "Abstract: electricity utility guidebook for geographic information systems data quality: metadata." Sep. https://www.epri.com/research/products/000000003002007921.

—. 2016a. "Common functions for DER group management, third edition." Nov. https://www.epri.com/research/products/000000003002008215.

FERC. 2020. Regional transmission organizations (RTO)/independent system operator (ISO). https://www.ferc.gov/industries/electric/indus-act/rto.asp.

GL, DNV. 2014. "A review of distributed energy resources." Sep. http://documents.dps.ny.gov/public/Common/ViewDoc.aspx?DocRefId=\%7B52BC75E8 -BBDD-4E2C-9D7D-34C160E0753A\%7D.

Greentech Media. 2017. The Distributed Energy Resource Management System Comes of Age. https://www.greentechmedia.com/articles/read/the-distributed-energy-resourcemanagement-system-comes-of-age.

GridWise Architecture Council. 2008. "GridWise Interoperability Context-Setting Framework."

—. 2015. "GridWise transactive energy framework, version 1.0." Jan. https://www.gridwiseac.org/pdfs/te framework_report pnnl-22946.pdf.

Gridworks. 2018. "The role of distributed energy resources in today's grid transition." Aug. https://gridworks.org/wp-content/uploads/2018/09/GridLab RoleOfDER online-1.pdf.

Guidehouse insights. 2020. Guidehouse Insights Leaderboard: DERMS Vendors. https://guidehouseinsights.com/reports/guidehouse-insights-leaderboard-derms-vendors.

Hawaiian Electric. 2014. "Fast demand response: be a clean, lean, green company." https://www.hawaiianelectric.com/documents/products_and_services/demand_response/ HECO Fast DR SALES Presentation 2014.pdf.

Holy Cross Energy. 2019. "Electric rate tariff adjustments distribution flexibility program optional." https://www.holycross.com/wp-content/uploads/2019/06/Electric-ServiceTariffs-Rules-and-Regulations-amended-14May2019-CLEAN_a.pdf\#page=38.

IEEE. 2007. "IEEE Guide for Monitoring, Information Exchange, and Control of Distributed Resources Interconnected with Electric Power Systems." IEEE Std 1547.3-2007 1-160.

Ikaheimo, Jussi, Corentin Evens, and Seppo Karkkainen. 2010. "DER Aggregator Business: the Finnish case." http://www.ece.hut.fi/enete/DER_Aggregator_Business_Finnish_Case.pdf.

International Renewable Energy Agency. 2019a. "Aggregators: innovation landscape brief." https://www.irena.org//media/Files/IRENA/Agency/Publication/2019/Feb/IRENA_Innovation_Aggregators_20 
19.PDF?la=en\&hash=EB86C1C86A7649B25050F57799F2C0F609894A012016/01/CEE

PR_WP_2016-001.pdf.

—. 2019b. "Innovation landscape for a renewable power future: solution VI aggregating

distributed energy resources for grid services." https://irena.org/-

/media/Files/IRENA/Agency/Topics/Innovation-and-

Technology/IRENA Landscape Solution 06.pdf?la=en\&hash=509F4C4D7C052104D8 97063EC6578901FA514184.

ISO New England. 2020. Administering the wholesale electricity markets. https://www.isone.com/about/what-we-do/three-roles/administering-markets.

Kristov, Lorenzo. 2014. "DSO and TSO roles and responsibilities in the decentralized energy future." Sep.

https://www.gridwiseac.org/pdfs/workshop 091014/kristov 091114 pres.pdf.

Li, D, W.-Y. Chiu, and H Sun. 2017. "Chapter 7-demand side management in microgrid control systems." Microgrid 203-230.

Lian, Jianming, H. Ren, Yannan Sun, K. Kalsi, Di Wu, and S. Widergren. 2017. Transactive system part II: analysis of two pilot transactive systems using foundational theory and metrics. PNNL.

Loutan, Clyde. 2015. "Briefing on the duck curve and current system conditions." Jul. http://www.caiso.com/Documents/Briefing_DuckCurve_CurrentSystemConditionsISOPresentation-July2015.pdf.

Melton, Ronald B., Kevin P. Schneider, Eric Lightner, Thomas E. McDermott, Poorva Sharma, Yingchen Zhang, Fei Ding, et al. 2018. "Leveraging Standards to Create an Open Platform for the Development of Advanced Distribution Applications." IEEE Access (IEEE) 6: 37361-37370.

Migden-Ostrander, Janine, John Shenot, Camille Kadoch, Max Dupuy, and Carl Linvill. 2018. "Enabling third-party aggregation of distributed energy resources." Feb. https://www.raponline.org/wpcontent/uploads/2018/04/enabling third_party_aggregation_distributed energy resource s2.pdf.

NERC. 2017. "Distribution energy resources: connection modeling and reliability considerations." Feb.

https://www.nerc.com/comm/Other/essntlrlbltysrvcstskfrcDL/Distributed_Energy_Resou rces Report.pdf.

New York ISO. 2017. "Distributed Energy Resources Market Design Concept Proposal." Dec. https://www.nyiso.com/documents/20142/1391862/Distributed-Energy-Resources-2017Market-Design-Concept-Proposal.pdf/122a815f-b767-e67f-0a8f-323e5489c2b1.

—. 2020. "Manual 2: ancillary service manual." May. https://www.nyiso.com/documents/20142/2923301/ancserv.pdf/df83ac75-c616-8c89c664-99dfea06fe2f.

Open EI. 2020. Definition: distribution management system. https://openei.org/wiki/Definition:Distribution_Management_System.

PJM. 2019. "PJM manual 11: energy \& ancillary services market operations." Dec. https://www.pjm.com/-/media/documents/manuals/m11.ashx?la=en.

—. 2020b. "PJM manual 18: PJM capacity market." May. https://www.pjm.com//media/documents/manuals/m18.ashx?la=en. 
—. 2020a. "PJM manual 36: system restoration." Jun. https://pjm.com/ /media/documents/manuals/m36.ashx.

PJM State \& Member Training Dept. 2015. "Reactive reserves and generator D-curves." Jan. https://www.pjm.com/-/media/training/nerc-certifications/gen-exammaterials/gof/20160104-reactive-reserves-and-d-curve.ashx?la=en.

Prinsloo, Gerro, Andrea Mammoli, and Robert Dobson. 2017. "Customer domain supply and load coordination: a case for smart villages and transactive control in rural off-grid microgrids." Energy 430-441.

Rahimi, Farrokh., and Sasan Mokhtari. 2014. "A New Distribution System Operator Construct." Open Access Technology International, Inc., https://www.gridwiseac.org/pdfs/workshop 091014/a new dist sys optr construct pap er.pdf.

Schneider Electric. 2019. "EcoStruxure DERMS: providing grid flexibility for all grid edge devices." Nov. https://download.schneiderelectric.com/files?p_Doc_Ref=DERMS_SS 11719.

ScienceDirect. 2020. Energy management system. https://www.sciencedirect.com/topics/engineering/energy-management-system.

Singaravelan, A., and M. Kowsalya. 2017. "A practical investigation on conservation voltage reduction for its efficiency with electric home appliances." Energy Procedia 117: 724730.

Sun, Y, and B Frew. 2021. Challenges and Research Priorities in United States Wholesale Electricity Markets. DOE GMLC.

Taft, Jeffrey D. 2017. DER telemetry communication architecture for ESOs, DSOs, and system operators. Nov. https://gridarchitecture.pnnl.gov/media/advanced/DER_Communication_Structure final GMLC.pdf.

Taft, Jeffrey D., and Angela Becker-Dippmann. 2015. "Grid architecture." Jan. https://gridarchitecture.pnnl.gov/media/white-papers/Grid\%20Architecture $\% 20 \% 20$ \%20DOE\%20QER.pdf.

Taft, Jeffrey D., and Jim Ogle. 2021. "Grid Architecture Guidance Specification for FASTDERMS."

Taft, Jeffrey D., Kristov, Lorenzo, and Paul De Martini. 2015. "A reference model for distribution grid control in the 21st century." Jul. https://www.pnnl.gov/main/publications/external/technical reports/PNNL-24463.pdf.

Taft, Jeffrey D. 2016. "Architectural Basis for Highly Distributed Transactive Power Grids: Frameworks, Networks, and Grid Codes."

Taft, Jeffrey D, Paul De Martini, and Lorenzo Kristov. 2015. "A Reference Model for Distribution Grid Control in the 21st Century."

Taft, Jeffrey D. 2016. Architectural Basis for Highly Distributed Transactive Power Grids: Frameworks, Networks, and Grid Codes. Pacific Northwest National Laboratory.

Taft, Jeffrey D. 2019. "Grid architecture." IEEE Power \& Energy Magazine 18-28.

Tan, Sen, Josep M. Guerrero, Peilin Xie, Renke Han, and Juan C. Vasquez. 2020. "Brief survey on attack detection methods for cyber-physical systems." IEEE System Journal 1-11.

Thomas, Sharon. 2018. Evolution of the distribution system \& the potential for distribution-level markets: a primer for state utility regulators. The National Association of Regulatory Utility Commissioners. 
U.S. Department of Energy. 2006. "Benefits of demand response in electricity markets and recommendations for achieving them." Feb.

https://www.energy.gov/sites/prod/files/oeprod/DocumentsandMedia/DOE_Benefits_of Demand Response_in_Electricity_Markets_and_Recommendations_for_Achieving_The m Report to Congress.pdf.

—. 2016. "Distribution automation: results from the smart grid investment grant program." Sep. https://www.energy.gov/sites/prod/files/2016/11/f34/Distribution\%20Automation\%20Su mmary\%20Report 09-29-16.pdf.

-. 2020b. Grid Architecture. https://gmlc.doe.gov/projects/1.2.1.

—. 2020a. "Grid modernization: metrics analysis (GMLC 1.1) - Flexibility." Apr. https://gmlc.doe.gov/resources/grid-modernization-metrics-analysis-gmlc1.1-flexibility.

—. 2017b. "Modern distribution grid, vol. II: advanced technology maturity assessment." Mar. https://gridarchitecture.pnnl.gov/media/Modern-Distribution-Grid_Volume-II_v1_1.pdf.

—. 2017a. "Quadrennial energy review: transforming the nation's electricity system: the second installment of the QER." Jan. https://www.energy.gov/sites/prod/files/2017/02/f34/Quadrennial\%20Energy\%20Review --Second\%20Installment $\% 20 \% 28$ Full\%20Report $\% 29$.pdf.

—. 2015b. "Quadrennial technology review 2015, chapter 3: enabling modernization of the electric power system - technology assessment, flexible and distributed energy resources." http://energy.gov/sites/prod/files/2015/09/f26/QTR2015-3D-Flexible-andDistributed-Energy 0.pdf.

—. 2015a. "Voices of experience: insights into advanced distribution management systems." Feb. https://www.energy.gov/sites/prod/files/2015/02/f19/Voices\%20of\%20Experience\%20\%20Advanced\%20Distribution\%20Management\%20Systems\%20February\%202015.pdf.

Widergren, Steven E., Jason Fuller, Cristina Marinovici, and Abhishek Somani. 2014. "Residential transactive control demonstration." ISGT. Washington, DC, USA: IEEE. 15.

Xcel Energy. 2019. "Demand-side management Annual status report." Apr. https://www.xcelenergy.com/staticfiles/xeresponsive/Company/Rates\%20\&\%20Regulations/Public\%20Service\%20Company\%202 018\%20DSM\%20Annual\%20Status\%20Report_FINAL.pdf.

—. 2016. "Peak partner rewards program." https://www.xcelenergy.com/staticfiles/xeresponsive/Programs \%20and\%20Rebates/Business/CO-Peak-Partner-Rewards-infosheet.pdf.

-. 2020. Renewable Energy Options - Residential. https://www.xcelenergy.com/programs and_rebates/residential_programs_and_rebates/re newable_energy_options_residential. 


\section{Appendix A}

\section{Entity Definition}

The common entities in distribution grids are briefly introduced and defined.

Advanced distribution management system (ADMS): an integrated software platform that provides a variety of comprehensive distribution grid management functions, such as distribution voltage management via volt/volt ampere reactive optimization, conservation voltage reduction, fault and outage management, peak demand management, and advanced functions and optimizations considering distributed energy resources (U.S. Department of Energy 2015a).

Aggregator: an entity or agent that aggregates a group of assets or customers to act as a single participant in power system and/or power markets (Burger, et al. 2016). Through aggregation, small-scale distributed energy resources (DERs) can behave like a sizeable resource from the point of view of the electric grid. An aggregator might present the assets under its control as a virtual power plant, where an aggregation of dispersed DERs operates like a traditional power plant (International Renewable Energy Agency 2019a).

Advanced metering infrastructure (AMI): typically refers to the measurement and collection system that consists of smart meters, communications networks, and information management systems. Smart meter reading data can be remotely accessed and delivered to utilities and customers every 15 minutes or even faster (U.S. Department of Energy 2017a).

Distributed energy resource management system (DERMS): a software-based solution that enhances the utility's network awareness and visibility into distributed energy resources (DERs) and offers a number of control functionalities, such as asset monitoring and control, scheduling and dispatch, power and voltage control, and resource valuation (U.S. Department of Energy 2015b). A DERMS can also be used to monitor and control DER aggregations, forecast DER capabilities, and communicate with other enterprise systems and into DER aggregators (EPRI 2016a). In some cases, this functionality is provided by an advanced distribution management system.

Distribution management system (DMS): an operational system that is capable of collecting data from sensors and measurement devices; monitoring and analyzing grid conditions; and controlling the distribution grid to increase efficiency, mitigate overloads, and optimize power flows (Open EI 2020) (U.S. Department of Energy 2017b).

Distribution operator (DO): an entity that manages and operates the distribution grid. Generally, power delivery service quality is maintained by the distribution operator through control systems, such as distribution management systems and outage management systems.

Distribution System Operator (DSO): an entity responsible for balancing supply and demand variations at the distribution level and linking the wholesale and retail market agents while maintaining the traditional role of the operator as a custodian for distribution system reliability (Rahimi and Mokhtari 2014). This is an evolving concept, and there is a wide spectrum of organizational models for DSOs (Black \& Veatch Management Consulting 2020).

Energy management system (EMS): an automated system that collects measurement data, monitors the performance of the transmission system and in some cases primary distribution substations, and controls and optimizes system operation through optimizations and contingency analysis (ScienceDirect 2020). 
The energy management system is the transmission system's analog to the distribution management system (U.S. Department of Energy 2017b).

Flexible resource scheduler: an entity proposed in this project that aggregates and manages all distributed energy resources in a distribution network to provide firm services in wholesale electricity markets and transmission system operations. The geographic scope of an instance of the flexible resource scheduler will be the feeders connected to one substation.

Geographic information system (GIS): a computer system for storing the geographic data of transmission and distribution grid equipment, maintaining the grid asset database, and displaying data on a map (EPRI 2016b).

Market operator: an independent organization to administer and organize wholesale electricity markets. In the United States, independent system operators typically administer electricity markets and power system planning and operation (ISO New England 2020). In this report, the term market operator denotes the department that manages electricity markets. Power system planning and operation is beyond the responsibility of a market operator.

Outage management system (OMS): a computer-aided system for power outage identification, outage alerts, and service restoration assistance.

Supervisory control and data acquisition (SCADA): for power distribution application, a system capable of collecting data from remote sensors and measurement devices, monitoring distribution system status, and performing control actions.

Scheduling coordinator: an entity that represents market participants such as generators and load-serving entities and settles all services and trades related to wholesale electricity markets (California ISO 2020a). In this project, the scheduling coordinator is defined as the upstream market coordinator of the applicable distribution system, meaning that all market bids of the flexible resource scheduler will be processed by the scheduling coordinator before entering wholesale electricity markets.

Transactive market: a scalable energy market/platform designed for coordinating local energy transactions among dispersed distributed energy resources (GridWise Architecture Council 2015). Similar to the concept of a conventional electricity market, a transactive market also uses the price as the core "value signal" for control.

Transmission system operator (TSO): an entity that coordinates, controls, and monitors the power transmission grid (FERC 2020). In the United States, the definition is similar to those of an independent system operator (ISO) and regional transmission operator (RTO). The core function of the TSO in a wholesale electricity market is to maintain the security and reliability of power system operation. In this regard, the TSO service is normally specified in guidelines as part of the electricity market (ENTSO-E 2020). In this report, we do not further distinguish a TSO from an ISO or an RTO. 


\section{Appendix B}

\section{Settlement Plans}

Some existing settlement plans used by utilities to recruit distributed energy resources (DERs) for participating in demand response programs and/or providing grid services are listed here (U.S. Department of Energy 2006).

1. Traditional price/rate program. In addition to existing rate plans - such as a fixed rate, time-of-use rate, or real-time pricing rate (if applicable)_DERs might require a rate reduction for agreeing to provide grid services such as demand response. Depending on the capacity and flexibility of DERs, customers could choose from a selection of rate programs to fit their needs. If the DERs successfully respond to utility dispatch orders, the subscribed rate programs should reduce the energy bills for these DER owners.

2. Net energy metering program. Customers with a sufficient capacity of DERs could feed power back to the grid when the generation capability is abundant (e.g., rooftop solar panels producing more power than the demand of the customer). The amount of energy that is fed back to the grid will reduce the net energy usage, resulting in a reduced energy procurement cost for the customer.

3. Energy credit/reward program. If DERs can be separately metered, DERs could subscribe to an energy credit/reward program for the amount of power generation. The credit can be applied to reduce the customers' energy bills, similar to the net energy metering program, or it can be saved for later use or trade to other customers. If the DER generation is renewable- - e.g., generated by rooftop solar panels - an additional carbon emission credit could apply to reward the customers for generating clean energy.

4. Buyback program. Instead of negotiating with DERs, utilities could post a price/reward for certain desired services. DERs that find the posted price acceptable can sign contracts with the utilities and agree to provide the specified service when requested to obtain the posted reward/compensation (U.S. Department of Energy 2006).

5. Customized bilateral contract. A customized bilateral contract offers flexible rate plans for DER owners to participate in a variety of grid services. The customized contract will specify the technical details of DERs, involved grid services, rate plans, and operating protocols.

As discussed in Section 5.3.2, the flexible resource scheduler offers two DER incentive subscription options: contractually obligated and transactive resources. We expect that the DER settlement plans will be narrowed to two options in the Federated Architecture for Secure and Transactive Distributed Energy Resource Management Solutions (FAST-DERMS): One is a bilateral contract that is widely used for DER aggregation, and the other is a real-time pricing scheme that will be implemented for transactive resources. The detailed settlement plan design will not be elaborated upon because it is not the focus of this FAST-DERMS architecture document. 


\section{Appendix C}

\section{Grid Services}

This section briefly reviews common transmission and distribution grid services and identifies several representative services that could be implemented through the Federated Architecture for Secure and Transactive Distributed Energy Resource Management Solutions (FAST-DERMS). More comprehensive studies of grid services, which include testing ${ }^{1}$ and valuation ${ }^{2}$ of grid services, were undertaken under GMLC Projects 1.2.4 and 1.4.2.

\section{Transmission Grid Services}

A transmission grid connects power generation plants with load centers through long-distance transmission infrastructure. Balancing power generation from power plants with the system power demand is the major factor that influences the operation and control of transmission grids; thus, most transmission grid services are designed to manage power generation and demand variation at different timescales.

Figure C. 1 shows the most common transmission grid services and the event speeds of these services (U.S. Department of Energy 2017a). Here, energy market participation is included as a grid service. ${ }^{3}$ At different timescales, different tools will be employed to resolve the demand-supply variation. Automatic control strategies such as inertial response will kick in at the subsecond level, whereas grid investment and expansion through capacity markets and transmission and distribution planning will ensure long-term balance between power supply and demand.

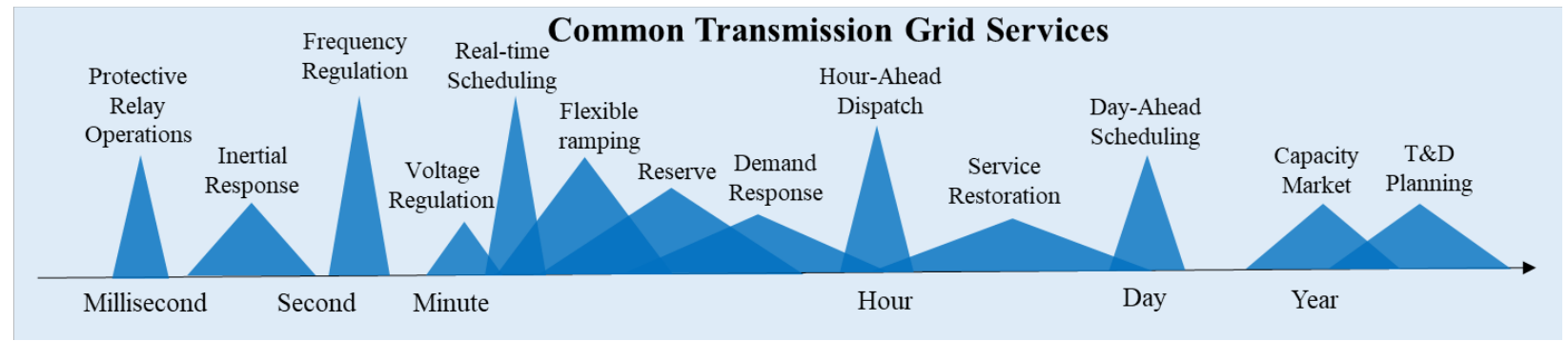

Figure C.1 Common bulk grid services and event speeds. Image from (U.S. Department of Energy 2017a)

Table C.1 distills the potential transmission grid services shown in Figure C.1 to those that FASTDERMS aims to provide, and it provides a brief review of these services. For each service, we summarize the technical requirements that are typically requested by transmission system operators and market operators from wholesale electricity market participants. The qualifying distributed energy resources (DERs) are identified as the controllable resources that can contribute such services.

\footnotetext{
${ }^{1}$ https://gmlc.doe.gov/projects/1.4.02.

2 L.C. Markel, S.W. Hadley, P.W. O'connor and A.K. Wolfe, "Valuation Framework for Informing Grid Modernization Decisions: Summary, A summary of the principles and process for valuing grid services and technologies," ORNL/SPR2019/1106, March 2019.

${ }^{3}$ The Federal Energy Regulatory Commission separates energy market operations and ancillary services. See the Energy Primer: A Handbook of Energy Market Basics, June 2020, available at https://www.ferc.gov/sites/default/files/2020-05/energyprimer.pdf.
} 
Table C.1 Review of Potential Bulk Grid Services for FAST-DERMS

\begin{tabular}{|c|c|c|c|}
\hline Service & Description & Typical Requirements & $\begin{array}{l}\text { Qualifying } \\
\text { DERs }\end{array}$ \\
\hline $\begin{array}{l}\text { Frequency } \\
\text { regulation }\end{array}$ & $\begin{array}{l}\text { Balances power generation with loads and } \\
\text { maintains system frequency at } 60 \mathrm{~Hz} \\
\text { (California ISO 2016) (New York ISO } \\
\text { 2020). The typical frequency regulation } \\
\text { signal, automatic generation control, is } \\
\text { updated every } 4 \text { seconds. }\end{array}$ & $\begin{array}{l}\text { Minimum aggregated } \\
\text { capacity }=0.5 \mathrm{MW} \\
\text { Minimum regulation capacity } \\
=0.1 \mathrm{MW} \\
\text { Service deployment } \\
<=4 \text { seconds }\end{array}$ & PV, BESS, EV \\
\hline $\begin{array}{l}\text { Voltage } \\
\text { regulation }\end{array}$ & $\begin{array}{l}\text { Maintains transmission voltages within } \\
\text { acceptable limits (New York ISO 2020) } \\
\text { (PJM State \& Member Training Dept. } \\
\text { 2015) }\end{array}$ & $\begin{array}{l}\text { Minimum sustained period }= \\
15 \text { minutes } \\
\text { Required to submit } \mathrm{P}-\mathrm{Q} \\
\text { curve }\end{array}$ & PV, BESS, EV \\
\hline $\begin{array}{l}\text { Real-time } \\
\text { scheduling } \\
\text { (real-time } \\
\text { energy market) }\end{array}$ & $\begin{array}{l}\text { Based on actual real-time operations and } \\
\text { enables participants to alter their day- } \\
\text { ahead bids (PJM 2019) (California ISO } \\
\text { 2020b). The typical real-time settlement } \\
\text { interval is } 5 \text { minutes. }\end{array}$ & $\begin{array}{l}\text { Minimum aggregated } \\
\text { capacity }=0.5 \mathrm{MW}\end{array}$ & PV, BESS, EV \\
\hline $\begin{array}{l}\text { Reserve } \\
\text { (spinning/non- } \\
\text { spinning) }\end{array}$ & $\begin{array}{l}\text { Provides backup generation capacity to } \\
\text { help maintain power balance and system } \\
\text { frequency (New York ISO 2020) }\end{array}$ & $\begin{array}{l}\text { Minimum aggregated } \\
\text { capacity }=0.5 \mathrm{MW} \\
\text { Service deployment } \\
<=10 \text { minutes } \\
\text { Minimum run time }=30 \\
\text { minutes }\end{array}$ & PV, BESS \\
\hline $\begin{array}{l}\text { Flexible } \\
\text { ramping }\end{array}$ & $\begin{array}{l}\text { Addresses the uncertainties of renewable } \\
\text { generation forecast (California ISO 2015a) } \\
\text { (California ISO 2015b). The typical } \\
\text { ramping service interval is } 5 \text { minutes. }\end{array}$ & $\begin{array}{l}\text { Minimum aggregated } \\
\text { capacity }=0.5 \mathrm{MW} \\
\text { Dispatch interval }<=5 \\
\text { minutes }\end{array}$ & PV, BESS \\
\hline $\begin{array}{l}\text { Day-ahead } \\
\text { scheduling } \\
\text { (day-ahead } \\
\text { energy market) }\end{array}$ & $\begin{array}{l}\text { Enables the participant to buy and sell } \\
\text { energy for the next operating day (PJM } \\
\text { 2019) (California ISO 2020b). The typical } \\
\text { day-ahead settlement interval is } 1 \text { hour. }\end{array}$ & $\begin{array}{l}\text { Minimum aggregated } \\
\text { capacity }=0.5 \mathrm{MW} \\
\text { Dispatch interval }<=1 \text { hour }\end{array}$ & $\begin{array}{l}\text { PV, BESS, EV, } \\
\text { building loads }\end{array}$ \\
\hline $\begin{array}{l}\text { Demand } \\
\text { response }\end{array}$ & $\begin{array}{l}\text { Employs demand curtailment to help } \\
\text { maintain system balance and reliability; } \\
\text { also known as emergency demand } \\
\text { response (GL 2014) (PJM 2019) }\end{array}$ & $\begin{array}{l}\text { Minimum aggregated } \\
\text { capacity }=0.5 \mathrm{MW} \\
\text { Minimum curtailment } \\
\text { capacity }=0.5 \mathrm{MW} \\
\text { Service deployment } \\
<=30 \text { minutes }\end{array}$ & $\begin{array}{l}\text { PV, BESS, EV, } \\
\text { building loads }\end{array}$ \\
\hline
\end{tabular}




\begin{tabular}{|l|l|l|l|}
\hline $\begin{array}{l}\text { Service } \\
\text { restoration } \\
\text { (black start) }\end{array}$ & $\begin{array}{l}\text { Generating units start up without relying } \\
\text { on external power and help crank other } \\
\text { generators and supply loads after a } \\
\text { blackout (PJM 2020a). }\end{array}$ & $\begin{array}{l}\text { Capability to self-start } \\
\text { without outside power } \\
\text { Capability to maintain } \\
\text { stability } \\
\text { Minimum run time }=16 \\
\text { hours }\end{array}$ & PV, BESS \\
\hline $\begin{array}{l}\text { Capacity } \\
\text { market }\end{array}$ & $\begin{array}{l}\text { Long-term product designed to ensure that } \\
\text { transmission systems have adequate } \\
\text { resources and capacity to meet reliability } \\
\text { requirements (PJM 2020b) }\end{array}$ & $\begin{array}{l}\text { Minimum aggregated } \\
\text { capacity }>=0.5 \text { MW }\end{array}$ & $\begin{array}{l}\text { PV, BESS, EV, } \\
\text { building loads }\end{array}$ \\
\hline
\end{tabular}

\section{Distribution Grid Services}

The distribution grid is responsible for delivering electricity from local substations to dispersed end users in a relatively small area compared to a transmission grid service area. Because the power supply-demand balance is primarily managed by generators and the transmission grid, distribution grids generally focus more on maintaining power delivery service quality. In this section, we emphasize distribution grid services in the operation and control stage because the long-term investment planning issues are similar to those in transmission grid services.

Traditional distribution grid control is based on three functions: power flow management, volt/volt ampere reactive (VAR) regulation, and demand response.

- Power flow management:

- The most significant feature of a distribution grid is its unbalanced three-phase power flow. Unbalanced power flows have negative impacts on power quality and equipment life span, and thus they should be closely monitored throughout the distribution grid. Power flow management has traditionally been accomplished through network reconfiguration, an approach that modifies distribution grid topology by operating switching devices (e.g., switches, circuit breakers, reclosers, and relays), most of which has been done manually. Network reconfiguration can be used to balance loads and regulate distribution voltages, and it is also an important solution to improving distribution grid reliability by reconnecting outage areas to other normal branches or feeders when faults happen. Load balancing service can also be provided by power electronic devices and reactive power controllers.

- An emerging, related topic is reverse power flow management. The fast-growing integration of DERs introduces reverse power flows in distribution grids, which could cause malfunctions of traditional protection relays. Reverse power flow management service can also be provided by switching devices.

- Voltage regulation:

- The voltage profile plays a key role in maintaining power delivery service quality. The service voltage magnitude must be kept within an acceptable range for all end users. Traditionally, voltage has been controlled through regulators and serial line drop compensators, some of which are automatic and some of which are manually adjusted. Reactive power has been mainly controlled by switching capacitors in and out of the circuit. More recently, integrated volt/VAR controls and optimizations have been used so that voltage regulators, on-load tap changers, capacitors, and other volt/VAR control devices (such as inverters) can be coordinated to 
maximize the improvement in distribution voltages while minimizing the switching operations of these devices (U.S. Department of Energy 2016) (Argonne National Laboratory 2017).

- Peak load management:

- Load management allows distribution utilities to reduce demand for electricity during peak usage time, which can in turn reduce costs by purchasing wholesale electricity at a lower price and improve distribution system reliability. Conservation voltage reduction is another important distribution voltage management application that reduces feeder voltage levels through on-load tap changers or through voltage regulators to reduce the total power consumption of the distribution grid. Conservation voltage reduction can also be regarded as a distribution grid peak load management service or a demand response service (Singaravelan and Kowsalya 2017).

Currently, distribution grid services are generally managed by distribution system operators (DSOs) using utility-owned or contracted devices, such as voltage regulators and large battery energy storage systems; thus, market-based financial rewards are typically not applicable to distribution grid services. Although providing distribution grid services might not bring revenue to DER owners, improving distribution grid operational flexibility and reliability will in turn benefit DER owners. In addition, some distribution utilities provide incentives in order to recruit DER customers to contribute to voltage and power management (Holy Cross Energy 2019). Also, distribution-level energy markets are an emerging topic that has already attracted attention from utilities (Thomas 2018); therefore, in this project, the DERs are expected to use their flexibility through FAST-DERMS to provide distribution grid services and to meet the control objectives of DSOs. 


\section{Appendix D}

\section{Specifications Listed in Reference Grid Architecture}

FAST-DERMS aligns with the following specifications that are listed in the reference grid architecture document (Taft and Ogle 2021):

“ 1.1 Distribution providers have a total Distribution System Operator role.

1.2 The DSO is responsible for managing distribution assets to meet an agreement with the TSO as to power and energy flows at the transmission/distribution interface substations.

1.3 The DSO is responsible for managing DER and power flows to/from and inside its distribution service area to maintain distribution reliability and meets its responsibilities to the TSO at the T/D interface, including power and energy flows across that interface, and management of volatility arising from distribution connected elements.

1.4 The TSO limits its grid observability to the Bulk Power System up to the T/D interface; it does not obtain grid state or operational data from DER devices or aggregators.

1.5 The TSO does not bypass the DSO to dispatch DER directly.

1.6 DER aggregators and other third-party energy services providers do not bypass the DSO to deal directly with the TSO.

2.3 Coordinate DER inverters with grid control to avoid conflicts at the distribution secondary level and to provide regulation service.

4.1 Use the Laminar Coordination Framework to develop specific Laminar Networks that correspond to the actual physical system being coordinated.

4.2 Follow the Framework to define logical information flows for the coordination process.

4.3 Maintain Laminar structure, even when DER are being coordinated via aggregators or other third parties.

4.4 Third parties can host Laminar nodes on behalf of the DER they aggregate.

4.5 Connect aggregators to the appropriate DSO to participate in the coordination data flows for the DER they manage in the service area of the DSO. Aggregators and other third parties may connect to more than one DSO if they have DER in more than one DSO service area (refer to Specification 1).

4.7 Use Laminar structure to define building-to-grid and microgrid-to-grid interfaces.

5.1 Use Laminar coordination structure to coordinate BTM and secondary-connected storage with other grid resources and devices. This applies to third party-operated storage as well as owner-operated storage.

5.2 BTM/secondary-connected storage coordination elements or functions must fit into a larger Laminar coordination framework so that storage coordination does not conflict with other distribution grid control devices and systems. 
5.3. If BTM/secondary-connected storage is to be used as a bulk power system resource, the coordination mechanism must not introduce coordination framework gapping or tier bypassing and must not enable hidden coupling, including via markets.

7.3 DER management must include access to real time circuit topology state, including grid fragment topology.

8.3 Provide communication connectivity that supports both device-to-control center communication and peer-to-peer communications, including Laminar Network coordination communications.

9.3 Consider the use of resilience as a cyber defense, instead of relying only on IT-type defenses.

10.2.2 Use structure for coordination and control that is consistent with Laminar Coordination Framework and logical energy networks (LENs).

10.2.3 Plan for faster action near the edge, i.e., at the segment level, with time frame of action slowing as one moves upward the structure.

10.2.4 Accommodate DER at any level in the structure.

10.3.1 Use Laminar structure for organizing EV charging.

10.3.2 Recognize the effect of transportation infrastructure networks (roadways) as related to the topology of the electric distribution system.

10.4.1 Sensing for distribution grid protection and control uses the observability platform structure and either the distributed intelligence platform structure or the Laminar coordination domain structure.

10.4.2 Sensors may be shared across multiple protection and control applications.

10.4.3 Direct sensor-to-local application data flows are permitted to provide low latency. Sensor data does not have to flow to a remote data store or broker first." 


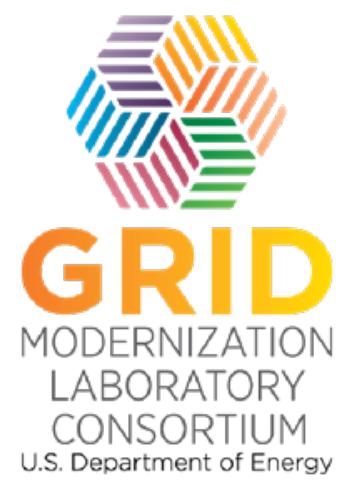

National Renewable Energy Laboratory 15103 Denver West Parkway

Golden, CO 80401

303-275-3000 • www.nrel.gov

https://gm/c.doe.gov 